\title{
Visualizing Orbital Content of Electronic Bands in Anisotropic 2D Semiconducting $\mathrm{ReSe}_{2}$
}

Byoung Ki Choi, ${ }^{1}$ Søren Ulstrup, ${ }^{2,3}$ Surani M. Gunasekera, ${ }^{4}$ Jiho Kim, ${ }^{5}$ Soo Yeon Lim, ${ }^{6}$ Luca Moreschini, ${ }^{3}$ Ji Seop Oh, ${ }^{3,7,8}$ Seung-Hyun Chun, ${ }^{9}$ Chris Jozwiak, ${ }^{3}$ Aaron Bostwick, ${ }^{3}$ Eli Rotenberg, ${ }^{3}$ Hyeonsik Cheong, ${ }^{6}$ In-Whan Lyo, ${ }^{5}$ Marcin Mucha-Kruczynski, ${ }^{4}$ and Young Jun Chang,,$^{*}$

${ }^{1}$ Department of Physics, University of Seoul, Seoul 02504, Republic of Korea

${ }^{2}$ Department of Physics and Astronomy, Aarhus University, Denmark, 8000 Aarhus C, Denmark

${ }^{3}$ Advanced Light Source (ALS), E. O. Lawrence Berkeley National Laboratory, Berkeley, California 94720, USA

${ }^{4}$ Centre for Nanoscience and Nanotechnology and Department of Physics, University of Bath, Bath BA2 7AY, United Kingdom

${ }^{5}$ Department of Physics, Yonsei University, Seoul, 03722, Republic of Korea

${ }^{6}$ Department of Physics, Sogang University, Seoul, 04107, Republic of Korea

${ }^{7}$ Center for Correlated Electron Systems, Institute for Basic Science (IBS), Seoul 08826, Republic of Korea

${ }^{8}$ Department of Physics and Astronomy, Seoul National University, Seoul 08826, Republic of Korea

${ }^{9}$ Department of Physics, Sejong University, Seoul 05006, Republic of Korea

*e-mail: yjchang@uos.ac.kr 


\section{ABSTRACT}

Many properties of layered materials change as they are thinned from their bulk forms down to single layers, with examples including indirect-to-direct band gap transition in $2 \mathrm{H}$ semiconducting transition metal dichalcogenides as well as thickness-dependent changes in the valence band structure in post-transition metal monochalcogenides and black phosphorus. Here, we use angle-resolved photoemission spectroscopy to study the electronic band structure of monolayer $\operatorname{ReSe}_{2}$, a semiconductor with a distorted 1T structure and in-plane anisotropy. By changing the polarization of incoming photons, we demonstrate that for $\mathrm{ReSe}_{2}$, in contrast to the $2 \mathrm{H}$ materials, the out-of-plane transition metal $d_{z}{ }^{2}$ and chalcogen $p_{z}$ orbitals do not contribute significantly to the top of the valence band which explains the reported weak changes in the electronic structure of this compound as a function of layer number. We estimate a band gap of $1.7 \mathrm{eV}$ in pristine $\mathrm{ReSe}_{2}$ using scanning tunneling spectroscopy and explore the implications on the gap following surface-doping with potassium. A lower bound of $1.4 \mathrm{eV}$ is estimated for the gap in the fully doped case, suggesting that doping-dependent many-body effects significantly affect the electronic properties of $\mathrm{ReSe}_{2}$. Our results, supported by density functional theory calculations, provide insight into the mechanisms behind polarizationdependent optical properties of rhenium dichalcogenides and highlight their place amongst two-dimensional crystals.

KEYWORDS: anisotropic 2D semiconductor, rhenium diselenide, orbital-selective electronic structure, transition metal dichalcogenides, two-dimensional materials. 
Among the plethora of two-dimensional (2D) materials entering the spotlight following the discovery of graphene, ${ }^{1}$ transition metal dichalcogenides (TMDs) attract huge interest as potential building blocks for innovative electronic and optoelectronic applications. ${ }^{2-4}$ TMDs, with their diverse and intriguing properties, such as semiconductivity, superconductivity, charge-density wave order, ferroelectricity, and ferromagnetism, ${ }^{2,4-10}$ open distinct device platforms as components in stacks of $2 \mathrm{D}$ crystals. As bulk materials are thinned down to monolayer (ML) forms to be used in such stacks, some of their properties might change due to quantum confinement effects. For example, semiconducting TMDs, such as $\mathrm{MX}_{2}(\mathrm{M}=\mathrm{Mo}, \mathrm{W}$ and $\mathrm{X}=\mathrm{S}, \mathrm{Se}$ ), exhibit an indirect electronic band gap in the bulk but a direct one in the ML limit, making the latter promising candidate materials for optoelectronics. This indirect-todirect transition as a function of decreasing layer number has been confirmed with various experimental methods, such as photoluminescence, ${ }^{4}$ ellipsometry, ${ }^{11}$ and angle-resolved photoelectron spectroscopy (ARPES). ${ }^{12}$ The strong layer-dependent evolution of the electronic structure indicates a significant interlayer coupling between individual layers.

At the same time, rhenium compounds $\left(\mathrm{ReS}_{2}\right.$ and $\left.\mathrm{ReSe}_{2}\right)$ are expected to undergo very small variations in the electronic band structure as a function of thickness due to weaker interlayer coupling than in other semiconducting TMDs. ${ }^{13-15}$ As opposed to the more common $1 \mathrm{~T}$ structure (Fig. 1a), $\operatorname{ReSe}_{2}$ is characterized by a $1 \mathrm{~T}^{\prime}$ structure with triclinic symmetry (space group: P1), ${ }^{16}$ induced by a Jahn-Teller-like structural distortion and seen as a zig-zag Re chain structure (red lines) in Fig. 1b. Such strong distortion induces anisotropy in the layer plane, resulting in, amongst others, strong linear dichroism. ${ }^{15,17}$ It also leads to larger interlayer spacing and introduces a large band gap into the electronic dispersion which would otherwise feature a half-filled metallic ground state. ${ }^{18}$ In contrast to semiconducting TMDs with $2 \mathrm{H}$ structure, the electronic band structure of $\mathrm{ReSe}_{2}$ and its orbital composition have not been well 
understood especially in the ML limit. $\mathrm{ReSe}_{2}$ shows nearly layer-independent photoluminescence signal, suggesting that a direct band gap is preserved for all thicknesses. ${ }^{19}$ Standard density functional theory (DFT) calculations predict an anisotropic valence band maximum (VBM) with heavy effective mass located slightly away from the $\Gamma$ point. When considering electron correlation effects, the VBM is rather uniform around the $\Gamma$ point, supporting the presence of a direct band gap in the ML. ${ }^{20}$ In case of bulk $\operatorname{ReSe}_{2}$, previous electronic structure studies found anisotropic 3D bulk bands with $\sim 0.1 \mathrm{eV}$ modulation along the $\mathrm{k}_{\mathrm{z}}$ direction driven by the interlayer interactions. ${ }^{21}$ In the similar compound $\operatorname{ReS}_{2}$, the 3D bulk band structure with in-plane anisotropy shifts the VBM away from the $\Gamma$ point along $\mathrm{k}_{\mathrm{z}}$, while ML and bilayer band structures indicate a direct band gap character with an increased effective hole mass in the ML. ${ }^{22,23}$ However, a direct experimental study of the electronic band structure of ML $\operatorname{ReSe}_{2}$, especially its orbital characteristics, from which one can understand the highly anisotropic carrier transport, optical, and vibrational properties, as well as interlayer interactions across the van der Waals spacing, is lacking. ${ }^{13,24-26}$

In this report, we study the electronic band structure of $\mathrm{ML} \mathrm{ReSe}_{2}$ which we grew epitaxially on a bilayer graphene (BLG)/SiC (0001) substrate by using molecular beam epitaxy (MBE). From ARPES measurements, we obtained the ML $\mathrm{ReSe}_{2}$ valence band structure and used polarization-resolved data as well as DFT calculations to elucidate the orbital contributions of Re to the valence band. We show that the out-of-plane transition metal $(\mathrm{Re}) d_{z^{2}}$ and chalcogen (Se) $p_{z}$ orbitals do not significantly contribute to states forming the VBM but rather to those in subbands located at higher binding energies $\left(E_{b i n}\right)$ in the range of $-2 \sim-2.5 \mathrm{eV}$. This is in contrast not only to $2 \mathrm{H}$ TMDs but also many other $2 \mathrm{D}$ materials, including post-transition metal monochalcogenides and black phosphorus, in which parts of the valence band are formed by states extending in the out-of-plane direction and are therefore strongly affected by interlayer 
coupling. ${ }^{27-33}$ As a result, the shape of the valence band in these materials depends sensitively on layer number, leading for example to indirect-to-direct band gap transitions. ${ }^{34}$ Our results provide a direct explanation of the relatively weak band structure changes in $\mathrm{ReSe}_{2}$ as a function of film thickness and emphasize the distinctness of rhenium compounds amongst not only the TMDs but also two-dimensional crystals in general. We also estimate the size of the band gap in our $\mathrm{ReSe}_{2}$ samples in both pristine and surface-doped cases, obtaining a gap of 1.7 $\mathrm{eV}$ in the pristine sample, while a lower bound of $1.4 \mathrm{eV}$ is found for fully doped $\mathrm{ReSe}_{2}$. The implications of these observations on many-body effects are discussed.

\section{RESULTS AND DISCUSSION}

Figure $1 \mathrm{~b}$ schematically shows the $\mathrm{ML} \mathrm{ReSe}_{2}$ on a BLG substrate. The crystal structure consists of a distorted hexagonal plane of Re atoms sandwiched between two corrugated Se layers, forming a $1 \mathrm{~T}^{\prime}$ structure with triclinic symmetry. The strong lattice distortion expands the unit cell (black dashed parallelograms) twice along both crystallographic directions in the plane, leading to the formation of zigzag chains of Re atoms (red lines), clearly distinct from the undistorted $1 \mathrm{~T}$ structure in Fig. 1a. The direction along the Re chains is roughly aligned with the zigzag direction of the underlying BLG on $6 \mathrm{H}-\mathrm{SiC}(0001)$, while the minimum lattice mismatch is estimated to be $1.6 \%$ for the match between $3 \mathrm{ReSe}_{2}$ unit cells and 8 graphene unit cells. Figure 1c shows the reflection high-energy electron diffraction (RHEED) pattern of the substrate. The BLG serves as an ideal substrate for fabricating van der Waals heterostructures and for studying their electronic structures due to the chemical inertness. ${ }^{5,12,35}$ The RHEED images in Fig. 1d show clear streaky lines indicating a well-ordered epitaxial growth of ML $\mathrm{ReSe}_{2}$ on top of the graphene lattice. Compared to the other ML $2 \mathrm{H}^{12,36-38}$ or $1 \mathrm{~T}$ phases ${ }^{39}$ showing $(1 \times 1)$ RHEED patterns, additional peaks (green arrows) indicate an increase of unit 
cell size due to strong lattice distortion of the $1 \mathrm{~T}^{\prime}$ phase, similar to the other $1 \mathrm{~T}^{\prime} \mathrm{TMDs}^{40}$ However, while in other TMDs the unit cell is often doubled due to a change of periodicity along only one of the in-plane directions, in $\operatorname{Re}$ dichalcogenides the unit cell area is approximately quadrupled as both in-plane primitive lattice vectors increase. Referring to the in-plane lattice constant of BLG $(\mathrm{a}=2.46 \AA)$, the in-plane lattice constants of $\mathrm{ReSe}_{2}$ are calculated to be $\sim 6.65 \pm 0.1 \AA$, similar to those of the bulk $\left(6.72 \AA\left(a_{1}\right)\right.$ and $\left.6.61 \AA\left(a_{2}\right)\right) .{ }^{16}$

In Fig. 1e, the Raman spectrum of our film shows sharp peaks corresponding to the reported peak positions (red circles) in $\operatorname{ReSe}_{2}$ flakes,${ }^{26}$ indicating the highly crystalline state of the film, comparable to the flakes. $\mathrm{ReSe}_{2}$ has a large number of Raman peaks, because the reduced crystal symmetry in the $1 \mathrm{~T}^{\prime}$ distorted structure splits the Raman peaks which are degenerate in the high-crystal symmetry 1T structures. Figure 1f shows a large-scale STM image with $\mathrm{ReSe}_{2}$ film coverage of $0.6 \mathrm{ML}$ on BLG (the corresponding RHEED pattern is shown in the inset of Fig. 1h). In Fig. $1 \mathrm{~g}$, the height of $\operatorname{ReSe}_{2}$ islands $(\sim 6.65 \AA)$ is consistent with the c-axis lattice constant of the bulk (6.72 $\AA$ ). In the zoomed-in STM image (Fig. 1h), the ML $\mathrm{ReSe}_{2}$ shows well-ordered crystalline phases along with noticeable stripe patterns, which indicate the anisotropic nature of the $1 \mathrm{~T}^{\prime}$ phase. Therefore, the $\mathrm{ML} \mathrm{ReSe}_{2}$ films are epitaxially grown on BLG with well-ordered crystalline and topographic quality. We also note that the randomly distributed lumps are probably selenium oxide or related amorphous components that originated during the selenium decapping procedure after the ex-situ sample transfer. 

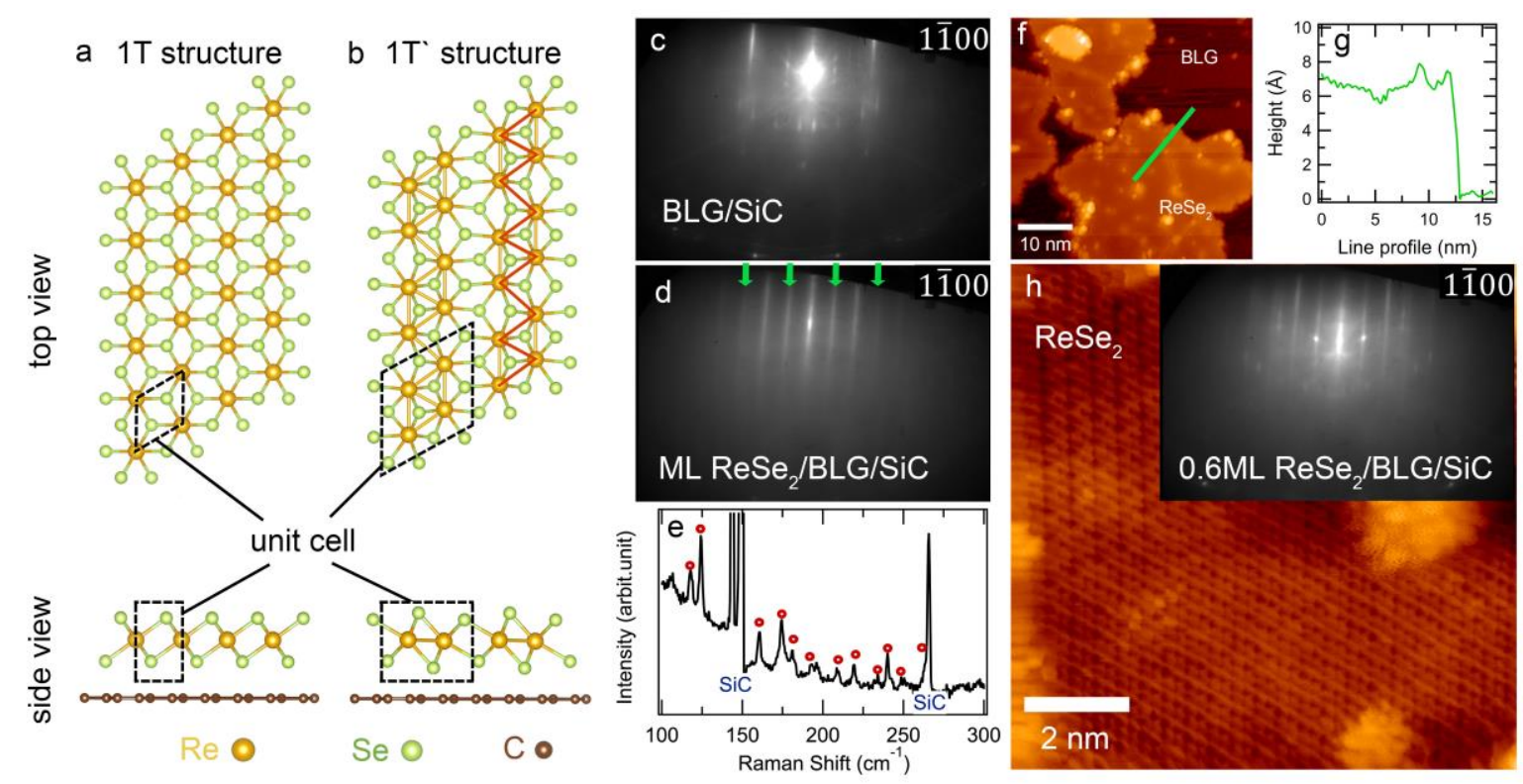

Figure 1. Morphology of ML ReSe 2 on BLG. $(a, b)$ Top- and side-view schematics of ML $\operatorname{ReSe}_{2}$ with $1 \mathrm{~T}$ and $1 \mathrm{~T}^{\prime}$ structures with graphene substrate added in the side view. The yellow, green, and brown balls represent the Re, Se, and $\mathrm{C}$ atoms, respectively and the red zigzag line shows the rhenium chains. (c,d) RHEED images of BLG and ML $\mathrm{ReSe}_{2}$, reflecting epitaxial growth of $\mathrm{ReSe}_{2}$ on BLG. The distortion in the $1 \mathrm{~T}^{\prime} \mathrm{ReSe}_{2}$ induces additional peaks (green arrows). (e) Raman spectrum of ML $\mathrm{ReSe}_{2}$ on BLG with sharp film peaks marked with red circles and two SiC substrate peaks. (f) Topographic STM image ( $\left.V_{\text {Sample }}=-2.0 \mathrm{~V}, I_{T}=50 \mathrm{pA}\right)$ of $0.6 \mathrm{ML}$ $\mathrm{ReSe}_{2}$ grown on BLG. (g) Line profile along the green line in f. (h) Magnified STM image $(-2.0 \mathrm{~V}, 50 \mathrm{pA})$ in the same region shows stripe patterns due to the anisotropic Re chain structure (Inset: RHEED image of corresponding 0.6 ML $\mathrm{ReSe}_{2}$ sample).

Figure 2 presents ARPES constant-energy (CE) maps of $1 \mathrm{ML} \mathrm{ReSe}_{2}$ grown on BLG at various $E_{b i n}$ obtained with p- and s-polarized light. Signal from the $\pi$ bands of BLG dominates near the graphene's Brillouin zone $(\mathrm{BZ})$ corner (marked as $\mathrm{K}_{\mathrm{G}}$ ) for both polarizations. Band structure of the $1 \mathrm{ML} \mathrm{ReSe} 2$ becomes evident only after saturating the graphene band. Because of the doubling of the in-plane lattice constants, the area of the $\mathrm{BZ}$ of $\mathrm{ReSe}_{2}$ is reduced to a quarter (black hexagons) as compared to the 1T phase (green dashed hexagon), which also explains the additional $(2 \times 2)$ RHEED patterns. The two-dimensionality of our film is evidenced by the photon-energy-independent band structure (see Fig. S1), to be contrasted with 
the dependence on photon energy observed across the 3D BZ of bulk $\operatorname{ReSe}_{2} \cdot{ }^{21,41}$ In addition, on a hexagonal graphene substrate, the $\mathrm{ReSe}_{2}$ islands can nucleate to form three energetically equivalent domains, each with the anisotropic crystal axis rotated by approximately $120^{\circ}$ with respect to each other, similar to $\mathrm{ReS}_{2} \cdot{ }^{42}$ Since the $\mathrm{ReSe}_{2}$ island size is about a few tens of nanometers in size (much smaller than the photon beam size of 20 50 micrometer), as shown in Fig. 1f, the ARPES intensity in the maps represents an average signal from all three anisotropic domains. This is why, although the BZ for a single crystal ML $\operatorname{ReSe}_{2}$ should be a slightly distorted regular hexagon, here we can work with an effective perfectly hexagonal BZ. Because of that, we use the labels $\mathrm{K}$ and $\mathrm{M}$ to denote any of the corners and centers of a side of a hexagonal BZ, respectively, rather than distinguish between different directions as would be necessary for a single domain. Finally, this explains why the CE maps reveal nearly isotropic electronic states around principal points ( $\mathrm{K}$ and $\mathrm{M}$ ), as shown in Fig. 2b,e (see Fig. S2), as opposed to the anisotropic valence bands of the bulk single crystals and flakes of $\operatorname{ReSe}_{2}$ and $\operatorname{ReS}_{2} \cdot{ }^{21,23,43}$ We note that similar multi-domain effects have been observed in ARPES maps of ML $1 \mathrm{~T}^{\prime} \mathrm{WTe}_{2}$ on BLG. ${ }^{40}$

The CE map at the Fermi level $\left(E_{F}\right)$ shows only background intensity at the $\Gamma$ point and signatures of graphene bands around all six $\mathrm{K}_{\mathrm{G}}$ (Fig. 2a,d), indicating that the $E_{F}$ is located inside the band gap of ML $\operatorname{ReSe}_{2}$. For $E_{\text {bin }}$ of $-1.1 \mathrm{eV}$, the electronic states emerge forming a nearly isotropic valence band at every $\Gamma$ point for both $p$ - and s-polarizations (Fig. 2b,e). From the intensity distribution in the CE map, we find that the VBM is centered at the $\Gamma$ point. Looking at energies below $E_{\text {bin }}=-1.1 \mathrm{eV}$, we can see enlarged valence band contours centered at the $\Gamma$ points as shown for $E_{b i n}$ of $-1.3 \mathrm{eV}$ in Fig. $2 \mathrm{c}, \mathrm{f}$. 

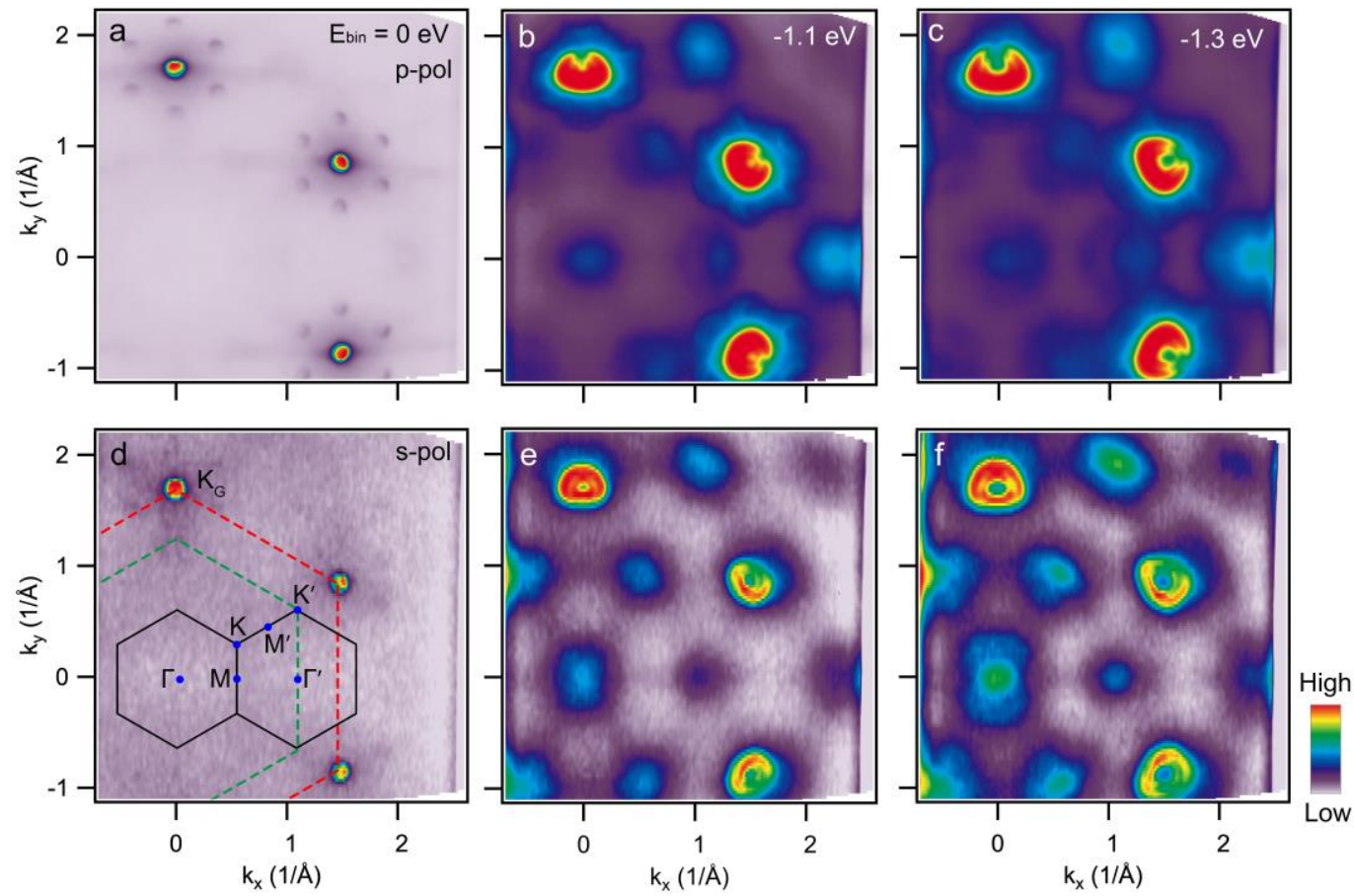

Figure 2. Constant energy ARPES maps of $1 T^{\prime} M L \operatorname{ReSe}_{2}$. (a-f) CE maps of ML $\operatorname{ReSe}_{2}$ with three selected $E_{\text {bin }}(=0,-1.1,-1.3 \mathrm{eV})$ with p-(a-c) and s-polarized (d-f) light $(h v=96 \mathrm{eV}, \mathrm{T}=$ $90 \mathrm{~K}$ ). The solid lines indicate the first and second Brillouin zones (BZs) of ML $\mathrm{ReSe}_{2}$ with $1 \mathrm{~T}^{\prime}$ structure. The green and red dashed lines correspond to the BZs of the undistorted 1T structure and BLG, respectively.

In Fig. 3a-d, the valence band dispersions along both $\Gamma-\mathrm{M}$ and $\Gamma-\mathrm{K}$ directions also show symmetric features centered at the $\Gamma$ point, for both $p$ - and s-polarization cases. In Fig. 3e, however, our DFT calculation (technical details of our computational method can be found in the density functional theory calculations part of the Methods section) of the ML $\operatorname{ReSe}_{2}$ band structure shows an anisotropic energy surface within the BZ (black hexagon), with a small dip at the $\Gamma$ point. When we take into account the multi-domain effect by overlapping band contours rotated by $120^{\circ}$ (red, yellow, and blue surfaces), as shown in Fig. 3f for the highest valence band, the calculated band structure becomes essentially 3-fold symmetric. The presence of a small dip at the $\Gamma$ point is not fully in agreement with the centered isotropic 
feature in our CE maps, possibly due to insufficient treatment of the electron-electron interactions. $^{20}$

The appearance of both the ML $\mathrm{ReSe}_{2} \mathrm{VBM}$ and BLG neutrality point in our ARPES data allows us to analyze the mutual alignment of the band structures of the two materials, shown as a schematic diagram in Fig. $3 \mathrm{~g}$. The neutrality point of BLG is positioned at the energy $E_{N}$ $=-0.33 \mathrm{eV}$ as seen in Fig. 3b,d, which is consistent with the range of $-0.24 \sim-0.32 \mathrm{eV}$ for a BLG on $n$-doped SiC. ${ }^{44,45}$ Using the results of work function measurements for BLG on $\mathrm{SiC},{ }^{44}$ we estimate the BLG neutrality point to be $4.54 \mathrm{eV}$ below the vacuum energy $\left(E_{v a c}\right)$. From our data fitted with gaussian functions (see Fig. S3 in the Supporting Information), the VBM of $\operatorname{ReSe}_{2}$ is situated $-1.1 \pm 0.05 \mathrm{eV}$ below $E_{F}$ and $0.77 \mathrm{eV}$ below the BLG neutrality point, see Fig. $3 \mathrm{~b}$ and $\mathrm{d}$. This places the $\mathrm{ReSe}_{2} \mathrm{VBM} 5.31 \mathrm{eV}$ below $E_{\text {vac }}$. At the same time, our DFT calculation predicts the VBM position in pristine $\mathrm{ReSe}_{2}$ as $5.24 \mathrm{eV}$ below $E_{v a c}$ (compared with an experimental value of $5.6 \mathrm{eV}$ for bulk $\left.\mathrm{ReSe}_{2}\right),{ }^{46}$ suggesting a minor Schottky barrier of $\lesssim 0.1$ $\mathrm{eV}$, similar to that deduced for $\mathrm{MoS}_{2}$ on graphene. ${ }^{47}$ Such a small barrier value implies a weak charge interaction between the $1 \mathrm{ML} \mathrm{ReSe}_{2}$ and BLG. In addition, we performed temperature dependent ARPES measurements between $80 \mathrm{~K}$ and $270 \mathrm{~K}$, as shown in Fig. S4. During the temperature cycle, we observe a $\sim 50 \mathrm{meV}$ energy shift, which likely results from the complex temperature dependent carrier densities in both $\mathrm{ReSe}_{2}$ and the SiC substrate. ${ }^{48}$ This small shift implies that the temperature-induced change of carrier density does not significantly affect the electronic structure of ML $\mathrm{ReSe}_{2}$ on BLG. We also note that all the ARPES measurements were performed at $80 \mathrm{~K}$, so that the temperature effect is minimal for understanding the ARPES results. 

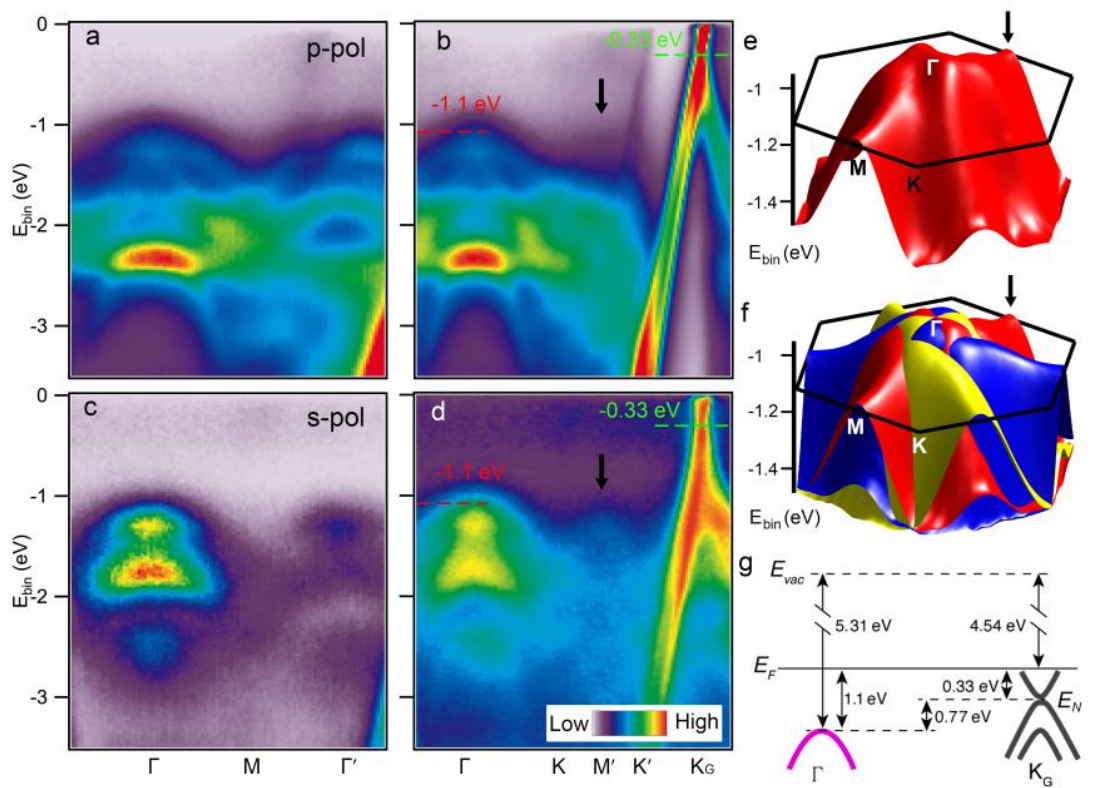

Figure 3. Valence band structure of ML ReSe 2 . (a-d) ARPES spectra along $\Gamma-\mathrm{M}-\Gamma^{\prime}$ and $\Gamma-\mathrm{K}-$ $\mathrm{M}^{\prime}$ for ML $\mathrm{ReSe}_{2}$ with the p-(a,b) and s-polarization (c,d). (e,f) Anisotropic energy surface within the first BZ (black hexagons) for a single domain (red, e) and for three domains related effectively by $120^{\circ}$ rotations (red, yellow, blue, f). The black arrows in (b) and (d) indicate the intensity close to the $\mathrm{M}^{\prime}$ point from electronic states of one of the domains as marked with the same arrows in e and $\mathrm{f}$ for the red surface. (g) Band alignment of pristine ML $\mathrm{ReSe}_{2}$ on BLG.

The orbital characteristics of the band structure is of great importance for understanding interlayer coupling and optical absorption processes. ${ }^{49,50}$ To identify the orbital contributions to the $\mathrm{ReSe}_{2}$ valence band, we compared ARPES spectra measured with p- and s-polarized light. The changes in the photocurrent intensity for the two polarizations are mostly due to the matrix element effects during the photoemission process, which encode the orbital character of the bands..$^{40,51,52}$ Within the dipole approximation, the electronic state in the crystal needs to be odd (even) with respect to reflection in the scattering plane in order to lead to a nonzero photocurrent for s-(p-) polarization. ${ }^{52,53}$ In Fig. 4a,b, the valence band spectra indeed show contrasting intensity distributions depending on light polarization. The distribution with p-(s-) polarized light is intense at $-2 \sim-2.5 \mathrm{eV}(-1 \sim-2 \mathrm{eV})$, respectively. While the presence of multiple domains makes it difficult to relate the orbitals to the scattering plane, two of them, 
the rhenium $d_{z}^{2}$ and selenium $p_{z}$ orbitals, have circular symmetry in the layer plane and hence couple to p-polarized photons. This suggests that the $d_{z}{ }^{2}$ and $p_{z}$ orbital states are concentrated deeper in the valence band rather than close to the VBM. We corroborate this with our calculations of orbital projections of the valence band states, Fig. 4c,d. These show that the $d_{z}^{2}$ and $p_{z}$ orbital states are concentrated near the lower part of the valence bands, while the rest of the d-orbitals $\left(d_{\mathrm{x}}^{2}-\mathrm{y}{ }^{2}\right.$ and $\left.t_{2 \mathrm{~g}}\right)$ form states in the upper part of the valence band, consistent with the previously suggested electronic structure for bulk $\mathrm{ReSe}_{2} \cdot{ }^{18,46} \mathrm{We}$ present orbital projections along the whole momentum range shown in Fig. 3a-d, which show qualitative agreement with the ARPES intensity, in the Supporting Information, Fig. S5. Our observation is also in agreement with the suggestion that the orbital makeup of valence band states in bulk $\operatorname{ReSe}_{2}$ is responsible for the observed negative pressure coefficients of its excitonic transitions ${ }^{54}$ In particular, the energy of the transition assigned to the $\mathrm{Z}$ point of the bulk BZ, which is projected on the $\Gamma$ point in the two-dimensional BZ of ML, undergoes the weakest changes with increasing pressure because the corresponding VBM states are formed mostly by orbitals less extended out of the plane than $d_{z}^{2}$ which are less affected by the applied pressure.

It is worth noting that in many layered materials including semiconducting $2 \mathrm{H}$ TMDs, posttransition metal monochalcogenides, and black phosphorus, the top valence band states are made of orbitals extending significantly out of the plane and responsible for interlayer interactions (mainly $d_{\mathrm{z}}{ }^{2}$ and $p_{\mathrm{z}}$ ). The lack of nearest neighbor layers in the case of ML crystals lowers the energy of such states while parts of the electronic dispersion made of in-plane orbitals remain largely unaffected. In the $2 \mathrm{H}$ semiconducting dichalcogenides, the transition metal $d_{z^{2}}$ and chalcogen $p_{z}$ orbitals form the top-most valence-band states around $\Gamma$ while the in-plane orbitals contribute to the states around K. With increasing number of layers, interlayer coupling splits the energies of the states at $\Gamma$ (which would be degenerate in the case of a stack 
of non-interacting layers), so that some of them increase in energy and overcome the local VBM at $\mathrm{K}$. This is accompanied by similar changes in the bottom conduction band where electronic states at $\mathrm{K}$, forming the conduction band minimum in the $\mathrm{ML}$, are overtaken in energy by states closer to $\Gamma$, resulting in a transition from a direct band gap in the ML to an indirect one in the bulk. ${ }^{12,34,55}$ Similar shift of the top valence band states dominated by contributions of $p_{z}$ orbitals leads to a significant decrease of electronic band gaps in the bulk as compared to the ML in monochalcogenides, such as, InSe, GaSe and GaS. ${ }^{27-33}$ However, in this group, an upwards shift of the states at $\Gamma$ in thicker crystals leads to an indirect-to-direct band gap from ML to bulk (with conduction band minimum always located at $\Gamma$ irrespectively of layer number). Black phosphorus, instead, remains a direct gap semiconductor for all thicknesses, with in-plane anisotropy leading to interesting photo-optoelectronic properties. ${ }^{56,57}$ As a result, the orbital composition of the valence band states, visualized in Fig. 4, makes rhenium dichalcogenides distinguished amongst the layered materials. This orbital composition is a consequence of the symmetry of the (undistorted) 1T structure and, more importantly, an additional valence electron of $\mathrm{Re}$ as compared to Mo or $\mathrm{W}$ forming $2 \mathrm{H}$ semiconducting TMDs. As a result of the latter, undistorted $1 \mathrm{~T} \operatorname{ReSe}_{2}$ would be a metal with several $d_{x^{2}-y^{2-}}$ and $d_{x y^{-}}$ character bands close to the $E_{F}{ }^{18}$ These $d$ orbitals lie in the layer plane and thus are strongly affected by any in-plane distortions which modify Re-Re couplings. The large distortion driving the shift from the $1 \mathrm{~T}$ to $1 \mathrm{~T}^{\prime}$ structure lowers the energy of the mentioned bands across the whole new, smaller BZ. The affected bands move down in energy but remain above other occupied bands and so form the top valence band of the now semiconducting $\operatorname{ReSe}_{2}$. Ultimately, this explains the weak crystal-thickness-dependence of the electronic band gap in $\operatorname{ReSe}_{2}$ and possibly also in $\operatorname{ReS}_{2} \cdot{ }^{13}$ 

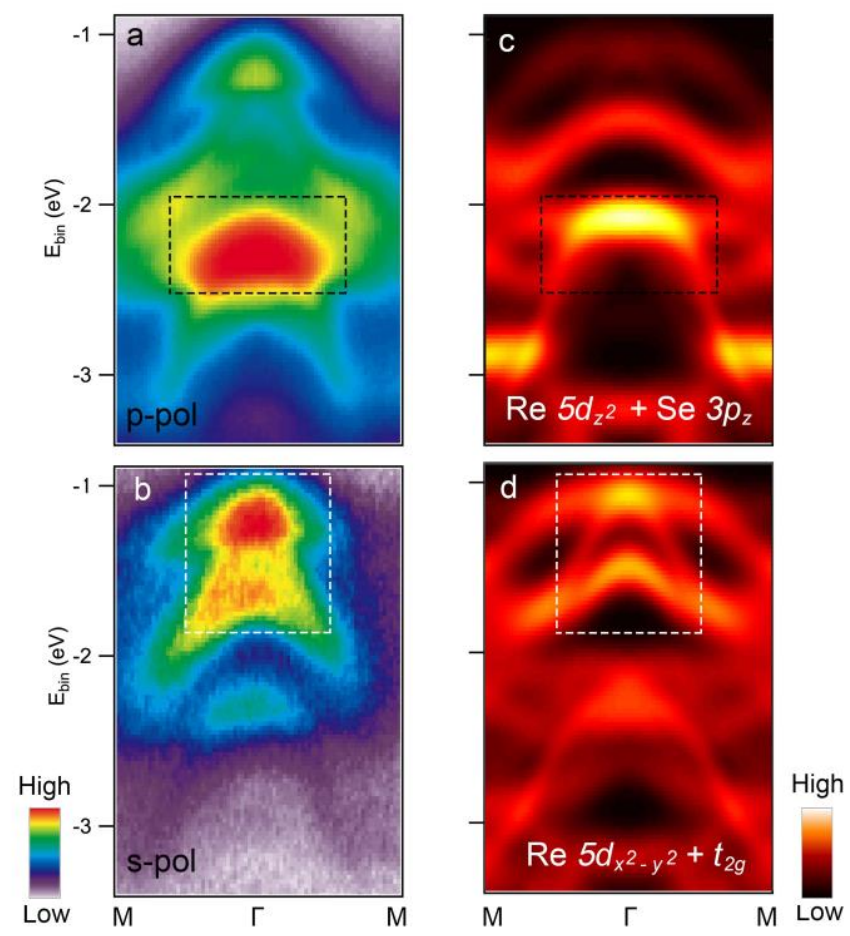

Figure 4. Orbital-selective valence band structure of $\mathrm{ML} \mathrm{ReSe}_{2}$. (a,b) Valence band dispersion along M- $\Gamma-\mathrm{M}$ for $p$ - (a) and $s$-polarized light (b). (c,d) Calculated density of states projections on the $\operatorname{Re} 5 d_{z}^{2}$ and $\operatorname{Se} 3 p_{z}$ (c) or the remaining Re $d$ orbitals (d). The black and white dashed boxes represent the intense distributions for the ARPES maps with each polarization, which are well accounted for by the calculated intensity profile for $d_{z}{ }^{2}+p_{z}$ or the sum of all other $d$ orbitals, respectively.

A detailed comparison of the ARPES data and our band structure calculation is presented in Fig. 5a-d, where the theoretical dispersion is plotted using red, yellow, and blue lines corresponding to the three different domains with colors to be compared to the surfaces in Fig. 3f. Overall, the calculated bands overlap well with the ARPES features. The relative rotations of the three domains lead to three distinctive energy dispersions. However, notice that for each of the surfaces in Fig. 3e,f, the dispersion along one of the $\mathrm{M}-\Gamma-\mathrm{M}$ directions is relatively flat while it crosses a steep hill in the perpendicular $\mathrm{K}-\Gamma-\mathrm{K}$ direction. The former corresponds to the direction perpendicular and the latter parallel to the Re chains. ${ }^{21}$ Because of the relative rotations, each of the domains contributes a dispersive feature along one of the $\Gamma-\mathrm{K}$ directions along its $\mathrm{K}-\mathrm{M}^{\prime}-\mathrm{K}^{\prime}$ segment and we have marked one of these with black arrows in the ARPES 
maps in Fig. 3b,d and 5b,d, as well as in Fig. 3e,f (backfolded into the first BZ). Together, the flat and dispersive behavior of the electronic bands implies that charges are localized (delocalized) along the direction perpendicular (parallel) direction to the Re chains, as reflected by nearly flat (dispersive) regions of the calculated top valence band drawn in yellow (red) in Fig. 5.
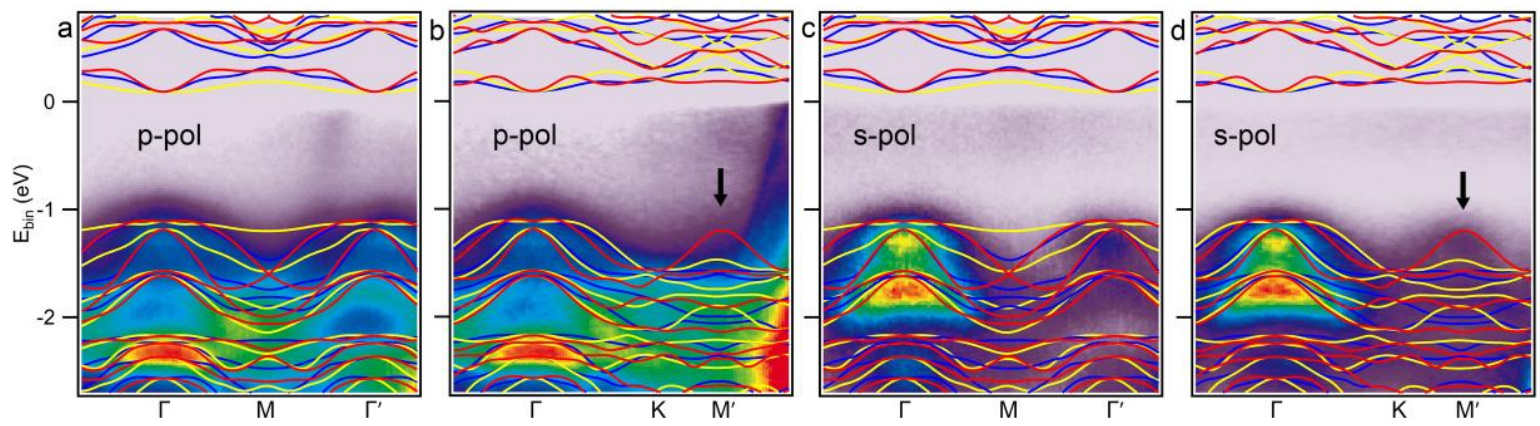

Figure 5. Comparison of DFT and ARPES bands of ML ReSe 2 . (a-d) Comparison of the ARPES data with p-/s-polarizations and our band structure calculations for the three rotated domains (red, yellow, and blue). The black arrows indicate the distinct dispersive states contributed by one of the domains (red).

To support our conclusion of only weak changes in the valence band structure of $\operatorname{ReSe}_{2}$ as a function of thickness, we further compare its valence band structure to that of a bilayer (2 ML). In Fig. 6a,b, we compare $(2 \times 2)$ RHEED patterns for 1 and $2 \mathrm{ML}$, where the main change in the $2 \mathrm{ML}$ case is an increased broadening of the streaks. As presented in Fig. 6c,d, the valence band structure of 2 ML does not exhibit an abrupt change of its features compared to the $1 \mathrm{ML}$, but displays a relatively small energy shift of $\sim 0.2 \mathrm{eV}$ for both the VBM and the intense subbands at higher $E_{b i n}$ around $\Gamma$, as indicated with dashed lines. Our experimental data is consistent with our DFT calculations which indicates little change in the valence band between $1 \mathrm{ML}$ and $2 \mathrm{ML} \mathrm{ReSe}$. This energy shift is consistent with the small change $(0.22 \mathrm{eV})$ of the optical band gap between $1 \mathrm{ML}$ and $2 \mathrm{ML} \mathrm{ReSe} 2$ flakes. ${ }^{19}$ Both the absence of an abrupt band 
structure change and the small band shift are in line with the weak thickness-dependence of the shape of the valence band in $\mathrm{ReSe}_{2}$. We note that the increased broadening of the ARPES intensity in the $2 \mathrm{ML}$ data is probably due to structural disorder in the second layer, similar to the broadened RHEED pattern.

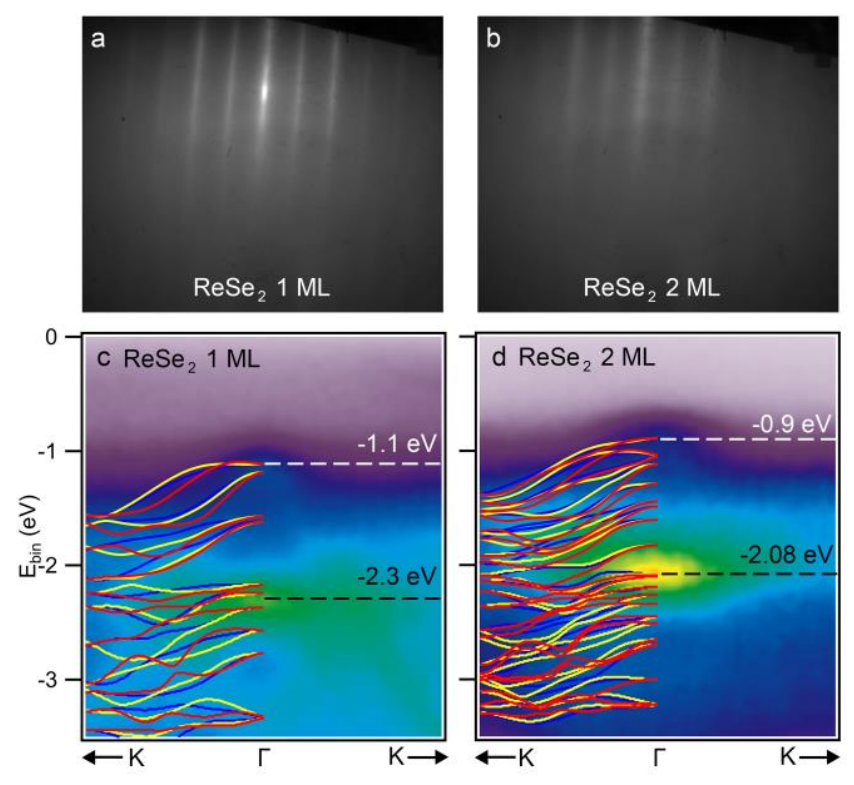

Figure 6. Comparison of $1 \mathrm{ML}$ and $2 \mathrm{ML} \mathrm{ReSe}_{2}$. (a,b) RHEED images of $1 \mathrm{ML}$ and $2 \mathrm{ML}$ $\mathrm{ReSe}_{2}$. The enhanced disorder and multi-domain effect may increase broadening in the $2 \mathrm{ML}$ sample. (c,d) ARPES bands with DFT calculations along K- $\Gamma-\mathrm{K}$ for $1 \mathrm{ML}$ (c) and $2 \mathrm{ML}$ $\mathrm{ReSe}_{2}(\mathrm{~d})$. White and black dashed lines indicate the energy positions of the VBM and an intense band towards higher $E_{b i n}$ at the $\Gamma$ point.

As a non-trivial semiconducting TMD, it is essential to estimate the band gap of ML $\mathrm{ReSe}_{2}$. Since ARPES only measures the occupied electronic states, we deposited potassium onto the sample surface during measurements in order to electron dope the structure and thereby attempt to shift the conduction bands below the $E_{F}$. The doping effect saturates at large potassium concentrations at which potassium simply accumulates on the surface without further electron donation (see Fig. S6). ${ }^{12,58,59}$ Figure 7a-c show valence band cuts and a maximum energy shift of $0.31 \mathrm{eV}$ (from $-1.1 \mathrm{eV}$ to $-1.41 \mathrm{eV}$ ) during the potassium-dosage until a coverage of $1.2 \mathrm{ML}$ 
was reached (we define $1 \mathrm{ML}$ coverage by tracking the appearance of an interface peak of potassium $3 p$ using X-ray photoemission spectroscopy, see Fig. S6). The evolution of energy dispersion curves at the $\Gamma$ point reveals a gradual energy shift of the valence bands, as shown in Fig. 7a-d. However, the surface-doping via potassium not only induces an energy shift of the $\operatorname{ReSe}_{2}$ bands $(0.31 \mathrm{eV})$, but also shifts the underlying BLG bands down by $0.2 \mathrm{eV}$ (see Fig. S7). Following the model of BLG from [ref 60], we calculated the 2D carrier density change in the BLG from the pristine state $\left(1.6 \times 10^{13} \mathrm{~cm}^{-2}, E_{N}=-0.33 \mathrm{eV}\right)$ to the case of a $1.2 \mathrm{ML}$ potassium coverage $\left(4.1 \times 10^{13} \mathrm{~cm}^{-2}, E_{N}=-0.53 \mathrm{eV}\right) .{ }^{60}$ Assuming comparable carrier doping for both the $\mathrm{ReSe}_{2}$ and BLG, we can then estimate the actual doping density of $2 \sim 3 \times 10^{13} \mathrm{~cm}^{-}$ ${ }^{2}$ for $1 \mathrm{ML}$ potassium coverage, equivalent to $0.04 \sim 0.06$ electrons per potassium atom.

Ultimately, we do not observe intensity from the $\mathrm{ReSe}_{2}$ conduction band states near the $E_{F}$ during potassium doping, as doping beyond 1.2 ML does not shift the bands of either $\mathrm{ReSe}_{2}$ or BLG any further, but enhances a broad background signal, probably due to the potassium adatoms accumulating on the surface. Our DFT calculation shows quite flat conduction bands, as seen in Fig. 5a-d, and hence a considerable electronic density of states which requires a large number of electrons to populate - one of the possible reasons why we do not fill the conduction band states. For this reason, we can use the VBM position of $-1.41 \pm 0.05 \mathrm{eV}$ as a lower bound on the magnitude of the electronic band gap, by assuming that the $E_{F}$ is close to the minimum energy of such flat conduction bands. Our lower bound estimate is similar to the optical band gap in ML $\operatorname{ReSe}_{2}(1.32 \sim 1.47 \mathrm{eV}),{ }^{14,19}$ but somewhat smaller than the gap of $\sim 1.7 \mathrm{eV}$, which we obtained from scanning tunneling spectroscopy (STS) measurements on our $\mathrm{ReSe}_{2} / \mathrm{BLG}$ heterostructure, as shown in Fig. 7e. The big difference between the optical and STS gap is explained by the large exciton binding energy (i.e. $460-680 \mathrm{meV}$ ) resulting from strong manybody interactions. ${ }^{61}$ The uncertainty of the STS gap may also arise from the tip-induced band 
bending owing to poor screening of electric fields at a semiconductor surface. ${ }^{62}$ On the other hand, while both ARPES and STS measure the quasi-particle band gap, the difference between the ARPES lower bound and the STS value likely occurs due to the fact that the increased carrier density significantly alters the dielectric screening and thus the Coulomb interaction in the system, which renormalizes the band gap. ${ }^{63-65}$ This renormalization of the gap can mainly be described in terms of a rigid band shift. In $\mathrm{ML} \mathrm{WSe}_{2}$, the observed decrease of the gap with respect to the value for the undoped structure was of the order of $0.5 \mathrm{eV}$ for doping of $\sim 10^{13}$ $\mathrm{cm}^{-2},{ }^{66,67}$ which is similar to the estimated additional density provided by potassium in our case, supporting the notion that at 1.2 ML potassium coverage we might be close to occupying the conduction band states. Note that in the ML limit, the reduction of interlayer screening increases the exciton binding energy while the dielectric screening from the underlying substrates becomes increasingly important. ${ }^{62}$ In addition, the strong interfacial electronic interaction also induces in-gap states and effectively reduces the STS gap in the case of semiconducting TMD-metal interfaces, as demonstrated for example for Au substrates. ${ }^{68-71}$ Finally, further investigation of hetero-interfaces between $\mathrm{ReSe}_{2}$ and other materials are of great interest due to possible electronic states related to moiré potentials and interface states. $^{61,68}$
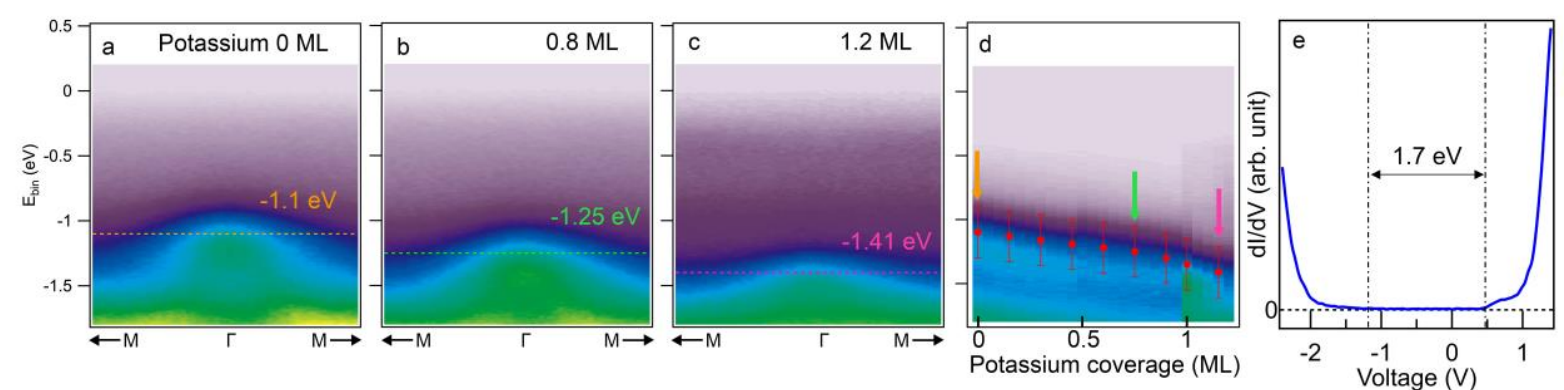

Figure 7. Band gap estimation of ML $\mathrm{ReSe}_{2}$. (a-c) Evolution of valence bands around the $\Gamma$ point with three potassium coverages of 0 (a), 0.8 (b) and 1.2 ML (c). (d) Shift of valence band maximum with potassium-coverage at $\Gamma$. Red circles indicate fitted band positions. The three arrows indicate the potassium coverage of a-c, respectively. (e) STS spectrum $\left(V_{\text {Sample }}=1.5 \mathrm{~V}\right.$, 
$I_{T}=100 \mathrm{pA}$ ) of pristine ML $\mathrm{ReSe}_{2}$ with estimated band gap of $1.7 \mathrm{eV}$.

\section{CONCLUSIONS}

In summary, we have successfully grown ML ReSe 2 epitaxially on BLG. We confirmed the single-crystalline properties of the ML-thick nanoscale $\mathrm{ReSe}_{2}$ islands by STM and Raman spectroscopy and resolved their valence band structure using ARPES. We also confirmed that, in contrast to many other two-dimensional crystals including the semiconducting $2 \mathrm{H}$ phases, i.e. $\mathrm{MoX}_{2}$ and $\mathrm{WX}_{2}(\mathrm{X}=\mathrm{S}, \mathrm{Se})$, monochalcogenides $\mathrm{InSe}, \mathrm{GaSe}$ and $\mathrm{GaS}$, and black phosphorus, in $\operatorname{ReSe}_{2}$ the $d_{z}{ }^{2}$ transition metal orbital contributes only weakly to the electronic states at the top of the valence band at $\Gamma$. Such orbital character as well as the lattice distortion significantly suppress interlayer interaction in this material. Our direct visualization of the orbital content of the valence band of $\operatorname{ReSe}_{2}$ with ARPES highlights the intriguing properties of this anisotropic 2D layered semiconductor with potential for future applications in electronic and optoelectronic devices. Finally, we provide a lower bound of $1.41 \mathrm{eV}$ on the electronic band gap in doped ML $\mathrm{ReSe}_{2}$ following potassium deposition and also an estimate of $1.7 \mathrm{eV}$ from STS in the pristine state. The different gap sizes highlight the significance of doping induced many-body effects in $\mathrm{ReSe}_{2}$.

\section{METHODS}

Molecular beam epitaxy. $\operatorname{ReSe}_{2}$ films were grown by using a home-built MBE chamber with a base pressure of $1 \times 10^{-10}$ Torr. We used $6 \mathrm{H}-\mathrm{SiC}(001)$ single crystal substrates, supplied by the Crystal Bank at Pusan National University. We annealed $6 \mathrm{H}-\mathrm{SiC}$ substrates at $1300^{\circ} \mathrm{C}$ in 2 min for 3 times in ultra-high vacuum (UHV) condition followed by the formation of BLG layer on the Si-terminated surface of SiC. We co-evaporated $\mathrm{Re}(99.97 \%)$ and $\mathrm{Se}(99.999 \%)$ by using 
an e-beam evaporator and an effusion cell, respectively. During film growth, we maintained a substrate temperature of $250^{\circ} \mathrm{C}$ for $10 \mathrm{~min}$ for $1 \mathrm{ML}$ thickness and then annealed the sample at $420^{\circ} \mathrm{C}$ for $30 \mathrm{~min}$. In case of $2 \mathrm{ML}$ sample, we extend the deposition duration accordingly. We carried out in situ RHEED measurements with a high voltage of $18 \mathrm{kV}$. For both STM and ARPES measurements, we covered the samples with an amorphous selenium layer at room temperature to protect the pristine surface from air exposure after the film growth. The samples were then annealed at $480 \mathrm{~K}$ in UHV to remove the selenium capping layer.

Raman spectroscopy. A $532 \mathrm{~nm}(2.33 \mathrm{eV})$ DPSS laser was used as an excitation source for Raman measurements. The laser beam was focused on the sample with a $\sim 1 \mu$ m diameter by a $50 \times$ objective lens $($ N.A. $=0.8)$ which also collected the scattered light from the sample. The scattered light was dispersed with a Jobin-Yvon Horiba iHR550 spectrometer (2400 grooves/mm) and was detected with a charge-coupled-device (CCD) using liquid nitrogen for cooling. We kept the laser power below $0.1 \mathrm{~mW}$ to avoid damages from local heating. The spectral resolution is $\sim 1 \mathrm{~cm}^{-1}$.

Scanning tunneling microscopy and spectroscopy. The experiments were performed in an UHV chamber $\left(3 \times 10^{-11} \mathrm{mbar}\right)$ by using a variable-temperature STM (OMICRON GmbH) equipped with home-built software and electronics. Electrochemically etched and Ar-sputtered tungsten tips were used. All STM and STS measurements were conducted at $79 \mathrm{~K}$. The bias voltages $\left(V_{\text {sample }}\right)$ stated in the topographic images were applied to the sample. For the STS spectrum, each $I(V)$ spectrum consists of 1024 data points and was Gaussian smoothed maintaining thermally limited energy resolution of $\Delta E=28 \mathrm{meV} . d I / d V$ spectrum was acquired by numerical differentiation of the $I-V$ sweep.

Angle resolved photoemission spectroscopy. ARPES measurements were performed in the micro-ARPES end-station (base pressure of $\sim 3 \times 10^{-11}$ Torr) at the MAESTRO facility at 
beamline 7.0.2 at the Advanced Light Source, Lawrence Berkeley National Laboratory, using p- or s-polarized photon. Depending on the polarization, the photoemission matrix element causes the intensity of the measured spectral features to vary strongly. ${ }^{72,73}$ The ARPES system was equipped with a Scienta R4000 electron analyzer. The lateral size of the synchrotron beam was estimated to be between 30 and $50 \mu \mathrm{m}$. The sample temperature was kept at $\sim 80 \mathrm{~K}$ during all the ARPES measurement. The total energy resolution was $20 \mathrm{meV}$ at $h v=96 \mathrm{eV}$ and calibrated with a polycrystalline gold film. A series of measurements was made with various photon energies in the range of $60-150 \mathrm{eV}$. Potassium-deposition was carried out by evaporation of potassium on the sample surfaces using a commercial SAES getter source mounted in the analysis chamber such that doping is performed in an optimum sample measurement position without moving the sample. Measurements and doping experiments were carried out at $90 \mathrm{~K}$. The amount of potassium deposited can be estimated from the potassium $3 p$ core level spectra (Supplementary Fig. S6a). The potassium $3 p$ peak initially appears at $E_{b i n}=\sim 18 \mathrm{eV}$ and grows in intensity as the initial potassium ML is completed. When the second potassium layer starts to grow, a chemically shifted potassium $3 p$ peak emerges at $E_{b i n}=\sim 19 \mathrm{eV} .{ }^{58,59} \operatorname{Re} 4 f$ core-level spectra shifts accordingly with the energy shift of $0.317 \mathrm{eV}$ upon reaching a potassium coverage of 1.2 ML (Supplementary Fig. S6).

Density functional theory calculations. DFT calculations used the Quantum ESPRESSO ${ }^{74,75}$ package and pseudopotentials from the PSlibrary ${ }^{76}$. For the band structure surfaces and cuts in Fig. 3 and Fig. 5, we used fully relativistic local density approximation (LDA) pseudopotentials, with the Perdew-Zunger ${ }^{77}$ parametrization of the exchange correlation energy. For the orbital projections along $\mathrm{M}-\Gamma-\mathrm{M}$ in Fig. 4, we used scalar-relativistic generalized gradient approximation (GGA) pseudopotentials (so that wave function projections onto atomic states classified only by orbital angular momentum could be conveniently obtained), with the 
Perdew-Burke-Ernzerhof ${ }^{78}$ parametrization of the exchange correlation energy. In all cases, the valence of Re was taken as 15 , and no van der Waals corrections were included. Results obtained using LDA/GGA scalar- and fully-relativistic pseudopotentials are compared in Ref. 26 and $41 .^{26,41}$ Atomic coordinates were taken from the Chemical Database Service ${ }^{79}$ and relaxed to obtain forces less than $0.006 \mathrm{eV} / \AA$. Kinetic energy cutoffs were typically, $60 \mathrm{Ry}$ $(816 \mathrm{eV})$ and Monkhorst-Pack ${ }^{80} \mathrm{k}$-point meshes of at least $8 \times 8 \times 1$ were used. To include the effect of different domains for Fig. 3f, we produced cuts along the three non-equivalent $\mathrm{K}-\Gamma$ $\mathrm{K}(\mathrm{M}-\Gamma-\mathrm{M})$ directions and plotted them together in such a way that the position of the left-most $\Gamma$ point corresponds to the $\Gamma$ point at $(0,0)$ for all the cuts.

\section{ASSOCIATED CONTENT}

Supporting Information

The Supporting Information is available free of charge on the ACS Publications website at DOI: Further characterization on the photon energy-dependent ARPES data, symmetric band structure along the three $\mathrm{M}-\Gamma-\mathrm{M}$ or $\mathrm{K}-\Gamma-\mathrm{K}$ directions, the valence band maximum and core level spectra during surface electron-doping, the temperature dependence, the orbital character of valence bands of ML $\mathrm{ReSe}_{2}$.

The authors declare no competing financial interests.

\section{AUTHOR INFORMATION}

\section{Corresponding Authors}

*E-mail: yjchang@uos.ac.kr. 


\section{Author Contributions}

B.K.C., and S.U. contributed equally. Y.J.C. conceived the experiments. B.K.C., S.-H.C., and Y.J.C. prepared thin films. J.K. and I.-W.L. carried out STM experiments. S.Y.L. and H.C. performed Raman measurements. B.K.C., S.U., L.M., J.O., C.J., A.B., E.R., and Y.J.C. performed ARPES measurements. S.M.G and M.M.-K. carried out theoretical calculations. B.K.C., S.U., M.M-K., and Y.J.C. prepared the manuscript. All authors discussed the results and commented on the manuscript.

\section{ACKNOWLEDGMENTS}

This work was supported by the National Research Foundation (NRF) grants funded by the Korean government (No. NRF-2020R1A2C200373211, 2019K1A3A7A09033389, and 2019R1A2C3006189) and made use of the Balena High Performance Computing (HPC) Service at the University of Bath. S.U. acknowledges financial support from the VILLUM FONDEN (Grant No. 15375). The Advanced Light Source is supported by the Director, Office of Science, Office of Basic Energy Sciences, of the U.S. Department of Energy under Contract No. DE-AC02-05CH11231. S.M.G. acknowledges support from the University of Bath and U.K. Engineering and Physical Sciences Research Council (EPSRC) through the Centre for Doctoral Training in Condensed Matter Physics, Grant EP/L015544/1EPSRC (UK). M.M.-K. was supported through the International Funding Scheme of the University of Bath. This work was supported by IBS-R009-D1. Authors thank B.L. Chittari and J. Kim for fruitful discussion.

\section{REFERENCES}

(1) Novoselov, K. S.; Mishchenko, A.; Carvalho, A.; Castro Neto, A. H. 2D Materials and van Der Waals Heterostructures. Science 2016, 353, 461.

(2) Mak, K. F.; Lee, C.; Hone, J.; Shan, J.; Heinz, T. F. Atomically Thin MoS2: A New 
Direct-Gap Semiconductor. Phys. Rev. Lett. 2010, 105, 136805.

(3) Xiong, Y.; Chen, H. W.; Zhang, D. W.; Zhou, P. Electronic and Optoelectronic Applications Based on ReS2. Phys. Status Solidi - Rapid Res. Lett. 2019, 13, 1800658.

(4) Splendiani, A.; Sun, L.; Zhang, Y.; Li, T.; Kim, J.; Chim, C. Y.; Galli, G.; Wang, F. Emerging Photoluminescence in Monolayer MoS 2. Nano Lett. 2010, 10, 1271-1275.

(5) Duvjir, G.; Choi, B. K.; Jang, I.; Ulstrup, S.; Kang, S.; Ly, T. T.; Kim, S.; Choi, Y. H.; Jozwiak, C.; Bostwick, A.; Rotenberg, E.; Park, J.; Sankar, R.; Kim, K.; Kim, J.; Chang, Y. J. Emergence of a Metal-Insulator Transition and High-Temperature Charge-Density Waves in VSe2 at the Monolayer Limit. Nano Lett. 2018, 18, 5432-5438.

(6) Xi, X.; Wang, Z.; Zhao, W.; Park, J. H.; Law, K. T.; Berger, H.; Forró, L.; Shan, J.; Mak, K. F. Ising Pairing in Superconducting NbSe2 Atomic Layers. Nat. Phys. 2016, 12, 139 143.

(7) Zhou, C.; Chai, Y. Ferroelectric-Gated Two-Dimensional-Material-Based Electron Devices. Adv. Electron. Mater. 2017, 3, 1600400.

(8) Huang, B.; Clark, G.; Navarro-Moratalla, E.; Klein, D. R.; Cheng, R.; Seyler, K. L.; Zhong, Di.; Schmidgall, E.; McGuire, M. A.; Cobden, D. H.; Yao, W.; Xiao, D.; JarilloHerrero, P.; Xu, X. Layer-Dependent Ferromagnetism in a van Der Waals Crystal down to the Monolayer Limit. Nature 2017, 546, 270-273.

(9) Gong, C.; Li, L.; Li, Z.; Ji, H.; Stern, A.; Xia, Y.; Cao, T.; Bao, W.; Wang, C.; Wang, Y.; Qiu, Z. Q.; Cava, R. J.; Louie, S. G.; Xia, J.; Zhang, X. Discovery of Intrinsic Ferromagnetism in Two-Dimensional van Der Waals Crystals. Nature 2017, 546, 265269.

(10) Watson, M. D.; Clark, O. J.; Mazzola, F.; Marković, I.; Sunko, V.; Kim, T. K.; Rossnagel, K.; King, P. D. C. Orbital- and Kz -Selective Hybridization of Se 4p and Ti 3d States in the Charge Density Wave Phase of TiSe2. Phys. Rev. Lett. 2019, 122, 076404.

(11) Li, Y.; Chernikov, A.; Zhang, X.; Rigosi, A.; Hill, H. M.; Zande, A. M. Van Der; Chenet, D. A.; Shih, E.; Hone, J.; Heinz, T. F. Measurement of the Optical Dielectric Function of Monolayer Transition-Metal Dichalcogenides : Phys. Rev. B 2014, 90, 205422.

(12) Zhang, Y.; Chang, T.; Zhou, B.; Cui, Y.; Yan, H.; Liu, Z.; Schmitt, F.; Lee, J.; Moore, R.; Chen, Y.; Lin, H.; Jeng, H.; Mo, S.; Hussain, Z.; Bansil, A.; Shen, Z. Direct Observation of the Transition from Indirect to Direct Bandgap in Atomically Thin Epitaxial MoSe 2. 2014, 9, 111-115.

(13) Tongay, S.; Sahin, H.; Ko, C.; Luce, A.; Fan, W.; Liu, K.; Zhou, J.; Huang, Y.; Ho, C.; Yan, J.; Ogletree, D. F.; Aloni, S.; Ji, J.; Li, S.; Li, J.; Peeters, F. M.; Wu, J. Monolayer Behaviour in Bulk ReS2 Due to Electronic and Vibrational Decoupling. Nat. Commun. 2014, 5, 3252.

(14) Liu, F.; Zheng, S.; Chaturvedi, A.; Zólyomi, V.; Zhou, J.; Fu, Q.; Zhu, C.; Yu, P.; Zeng, Q.; Drummond, N. D.; Fan, H. J.; Kloc, C.; Fal'ko, V. I.; He, X.; Liu, Z. Optoelectronic Properties of Atomically Thin ReSSe with Weak Interlayer Coupling. Nanoscale 2016, 8, 5826-5834.

(15) Arora, A.; Noky, J.; Drüppel, M.; Jariwala, B.; Deilmann, T.; Schneider, R.; Schmidt, R.; Del Pozo-Zamudio, O.; Stiehm, T.; Bhattacharya, A.; Krüger, P.; Michaelis de Vasconcellos, S.; Rohlfing, M.; Bratschitsch, R. Highly Anisotropic In-Plane Excitons in Atomically Thin and Bulklike 1T'-ReSe2. Nano Lett. 2017, 17, 3202-3207.

(16) Alcock, N. W.; Kjekshus, A. The Crystal Structure of Rese2. ACTA Chem. Scand. 1965, 19, 79-94. 
(17) Zhang, E.; Wang, P.; Li, Z.; Wang, H.; Song, C.; Huang, C.; Chen, Z. G.; Yang, L.; Zhang, K.; Lu, S.; Wang, W.; Liu, S.; Fang, H.; Zhou, X.; Yan, H.; Zou, J.; Wan, X.; Zhou, P.; Hu, W.; Xiu, F. Tunable Ambipolar Polarization-Sensitive Photodetectors Based on High-Anisotropy ReSe2 Nanosheets. ACS Nano 2016, 10, 8067-8077.

(18) Kertesz, M.; Hoffmann, R. Octahedral vs. Trigonal-Prismatic Coordination and Clustering in Transition-Metal Dichalcogenides. J. Am. Chem. Soc. 1984, 106, 34533460 .

(19) Zhao, H.; Wu, J.; Zhong, H.; Guo, Q.; Wang, X.; Xia, F.; Yang, L. Interlayer Interactions in Anisotropic Atomically Thin Rhenium Diselenide. Nano Res. 2015, 8, 3651.

(20) Zhong, H. X.; Gao, S.; Shi, J. J.; Yang, L. Quasiparticle Band Gaps, Excitonic Effects, and Anisotropic Optical Properties of the Monolayer Distorted 1T Diamond-Chain Structures ReS2 and ReSe2. Phys. Rev. B - Condens. Matter Mater. Phys. 2015, 92, 115438.

(21) Hart, L. S.; Webb, J. L.; Dale, S.; Bending, S. J.; Mucha-Kruczynski, M.; Wolverson, D.; Chen, C.; Avila, J.; Asensio, M. C. Electronic Bandstructure and van Der Waals Coupling of ReSe2 Revealed by High-Resolution Angle-Resolved Photoemission Spectroscopy. Sci. Rep. 2017, 7, 5145.

(22) Biswas, D.; Ganose, A. M.; Yano, R.; Riley, J. M.; Bawden, L.; Clark, O. J.; Feng, J.; Collins-Mcintyre, L.; Sajjad, M. T.; Meevasana, W.; Kim, T. K.; Hoesch, M.; Rault, J. E.; Sasagawa, T.; Scanlon, D. O.; King, P. D. C. Narrow-Band Anisotropic Electronic Structure of ReS2. Phys. Rev. B 2017, 96, 085205.

(23) Gehlmann, M.; Aguilera, I.; Bihlmayer, G.; Nemšák, S.; Nagler, P.; Gospodarič, P.; Zamborlini, G.; Eschbach, M.; Feyer, V.; Kronast, F.; Młyńczak, E.; Korn, T.; Plucinski, L.; Schüller, C.; Blügel, S.; Schneider, C. M. Direct Observation of the Band Gap Transition in Atomically Thin ReS2. Nano Lett. 2017, 17, 5187-5192.

(24) Lee, B. C.; Na, J.; Choi, J. H.; Ji, H.; Kim, G. T.; Joo, M. K. Probing Distinctive Electron Conduction in Multilayer Rhenium Disulfide. Adv. Mater. 2019, 31, 1805860.

(25) Aslan, O. B.; Chenet, D. A.; Van Der Zande, A. M.; Hone, J. C.; Heinz, T. F. Linearly Polarized Excitons in Single- and Few-Layer ReS2 Crystals. ACS Photonics 2016, 3, 96-101.

(26) Wolverson, D.; Crampin, S.; Kazemi, A. S.; Ilie, A.; Bending, S. J. Raman Spectra of Monolayer, Few-Layer, and Bulk ReSe2: An Anisotropic Layered Semiconductor. ACS Nano 2014, 8, 11154-11164.

(27) Bandurin, D. A.; Tyurnina, A. V.; Yu, G. L.; Mishchenko, A.; Zólyomi, V.; Morozov, S. V.; Kumar, R. K.; Gorbachev, R. V.; Kudrynskyi, Z. R.; Pezzini, S.; Kovalyuk, Z. D.; Zeitler, U.; Novoselov, K. S.; Patanè, A.; Eaves, L.; Grigorieva, I. V.; Fal'Ko, V. I.; Geim, A. K.; Cao, Y. High Electron Mobility, Quantum Hall Effect and Anomalous Optical Response in Atomically Thin InSe. Nat. Nanotechnol. 2017, 12, 223.

(28) Ben Aziza, Z.; Pierucci, D.; Henck, H.; Silly, M. G.; David, C.; Yoon, M.; Sirotti, F.; Xiao, K.; Eddrief, M.; Girard, J.-C.; Ouerghi, A. Tunable Quasiparticle Band Gap in Few-Layer GaSe/Graphene van Der Waals Heterostructures. Phys. Rev. B 2017, 96, 035407.

(29) Su Jung, C.; Shojaei, F.; Park, K.; Young Oh, J.; Soon Im, H.; Myung Jang, D.; Park, J.; Seok Kang, H. Red-to-Ultraviolet Emission Tuning of Two-Dimensional Gallium Sulfide/Selenide. ACS Nano 2015, 9, 9585.

(30) Shevitski, B.; Ulstrup, S.; Koch, R. J.; Cai, H.; Tongay, S.; Moreschini, L.; Jozwiak, C.; Bostwick, A.; Zettl, A.; Rotenberg, E.; Aloni, S. Tunable Electronic Structure in Gallium 
Chalcogenide van Der Waals Compounds. Phys. Rev. B 2019, 100, 165112.

(31) Rybkovskiy, D. V.; Osadchy, A. V.; Obraztsova, E. D. Transition from Parabolic to Ring-Shaped Valence Band Maximum in Few-Layer GaS, GaSe, and InSe. Phys. Rev. B 2014, 90, 235302.

(32) Hamer, M.; J.; Zultak, J.; V. Tyurnina, A.; Zólyomi, V.; Terry, D.; Barinov, A.; Garner, A.; Donoghue, J.; P. Rooney, A.; Kandyba, V.; Giampietri, A.; Graham, A.; Teutsch, N.; Xia, X.; Koperski, M.; J. Haigh, S.; I. Fal'ko, V.; V. Gorbachev, R.; R. Wilson, N. Indirect to Direct Gap Crossover in Two-Dimensional InSe Revealed by AngleResolved Photoemission Spectroscopy. ACS Nano 2019, 13, 2136.

(33) Magorrian, S. J.; Zólyomi, V.; Fal'ko, V. I. Electronic and Optical Properties of TwoDimensional InSe from a DFT-Parametrized Tight-Binding Model. Phys. Rev. B 2016, 94, 245431.

(34) Jin, W.; Yeh, P. C.; Zaki, N.; Zhang, D.; Sadowski, J. T.; Al-Mahboob, A.; Van Der Zande, A. M.; Chenet, D. A.; Dadap, J. I.; Herman, I. P.; Sutter, P.; Hone, J.; Osgood, R. M. Direct Measurement of the Thickness-Dependent Electronic Band Structure of MoS2 Using Angle-Resolved Photoemission Spectroscopy. Phys. Rev. Lett. 2013, 111, 106801.

(35) Miwa, J. A.; Dendzik, M.; Grønborg, S. S.; Bianchi, M.; Lauritsen, J. V.; Hofmann, P.; Ulstrup, S. Van Der Waals Epitaxy of Two-Dimensional MoS2-Graphene Heterostructures in Ultrahigh Vacuum. ACS Nano 2015, 9 (6), 6502-6510.

(36) Yue, R.; Barton, A. T.; Zhu, H.; Azcatl, A.; Pena, L. F.; Wang, J.; Peng, X.; Lu, N.; Cheng, L.; Addou, R.; McDonnell, S.; Colombo, L.; Hsu, J. W. P.; Kim, J.; Kim, M. J.; Wallace, R. M.; Hinkle, C. L. HfSe2 Thin Films: 2D Transition Metal Dichalcogenides Grown by Molecular Beam Epitaxy. ACS Nano 2015, 9, 474-480.

(37) Tsoutsou, D.; Aretouli, K. E.; Tsipas, P.; Marquez-Velasco, J.; Xenogiannopoulou, E.; Kelaidis, N.; Aminalragia Giamini, S.; Dimoulas, A. Epitaxial 2D MoSe2 (HfSe2) Semiconductor/2D TaSe2 Metal van Der Waals Heterostructures. ACS Appl. Mater. Interfaces 2016, 8, 1836-1841.

(38) Tsipas, P.; Tsoutsou, D.; Marquez-Velasco, J.; Aretouli, K. E.; Xenogiannopoulou, E.; Vassalou, E.; Kordas, G.; Dimoulas, A. Epitaxial ZrSe2/MoSe2 Semiconductor v.d. Waals Heterostructures on Wide Band Gap AlN Substrates. Microelectron. Eng. 2015, 147, 269-272.

(39) Chen, P.; Chan, Y. H.; Fang, X. Y.; Zhang, Y.; Chou, M. Y.; Mo, S. K.; Hussain, Z.; Fedorov, A. V.; Chiang, T. C. Charge Density Wave Transition in Single-Layer Titanium Diselenide. Nat. Commun. 2015, 6, 8943.

(40) Tang, S.; Zhang, C.; Wong, Di.; Pedramrazi, Z.; Tsai, H. Z.; Jia, C.; Moritz, B.; Claassen, M.; Ryu, H.; Kahn, S.; Jiang, J.; Yan, H.; Hashimoto, M.; Lu, D.; Moore, R. G.; Hwang, C. C.; Hwang, C.; Hussain, Z.; Chen, Y.; Ugeda, M. M.; Liu, Z.; Xie, X.; Devereaux, T. P.; Crommie, M. F.; Mo, S. K.; Shen, Z. X. Quantum Spin Hall State in Monolayer 1T'WTe 2. Nat. Phys. 2017, 13, 683-687.

(41) Gunasekera, S. M.; Wolverson, D.; Hart, L. S.; Mucha-Kruczynski, M. Electronic Band Structure of Rhenium Dichalcogenides. J. Electron. Mater. 2018, 47, 4314-4320.

(42) Wu, K.; Chen, B.; Yang, S.; Wang, G.; Kong, W.; Cai, H.; Aoki, T.; Soignard, E.; Marie, X.; Yano, A.; Suslu, A.; Urbaszek, B.; Tongay, S. Domain Architectures and Grain Boundaries in Chemical Vapor Deposited Highly Anisotropic ReS2 Monolayer Films. Nano Lett. 2016, 16, 5888-5894.

(43) Webb, J. L.; Hart, L. S.; Wolverson, D.; Chen, C.; Avila, J.; Asensio, M. C. Electronic 
Band Structure of ReS2 by High-Resolution Angle-Resolved Photoemission Spectroscopy. Phys. Rev. B 2017, 96, 115205.

(44) Mammadov, S.; Ristein, J.; Krone, J.; Raidel, C.; Wanke, M.; Wiesmann, V.; Speck, F.; Seyller, T. Work Function of Graphene Multilayers on SiC(0001). 2D Mater. 2017, 4, 015043.

(45) Ohta, T.; Bostwick, A.; Seyller, T.; Horn, K.; Rotenberg, E. Controlling the Electronic Structure of Bilayer Graphene. Science 2006, 313, 951.

(46) Ho, C. H.; Huang, Y. S.; Chen, J. L.; Dann, T. E.; Tiong, K. K. Electronic Structure of $\mathrm{ReS} 2$ and ReSe2 from First-Principles Calculations, Photoelectron Spectroscopy, and Electrolyte Electroreflectance. Phys. Rev. B 1999, 60, 15766.

(47) Coy Diaz, H.; Avila, J.; Chen, C.; Addou, R.; Asensio, M. C.; Batzill, M. Direct Observation of Interlayer Hybridization and Dirac Relativistic Carriers in Graphene/MoS2 van Der Waals Heterostructures. Nano Lett. 2015, 15, 1135-1140.

(48) Ristein, J.; Mammadov, S.; Seyller, T. Origin of Doping in Quasi-Free-Standing Graphene on Silicon Carbide. Phys. Rev. Lett. 2012, 108, 246104.

(49) Wang, G.; Robert, C.; Suslu, A.; Chen, B.; Yang, S.; Alamdari, S.; Gerber, I. C.; Amand, T.; Marie, X.; Tongay, S.; Urbaszek, B. Spin-Orbit Engineering in Transition Metal Dichalcogenide Alloy Monolayers. Nat. Commun. 2015, 6, 10110.

(50) Manchon, A.; Koo, H. C.; Nitta, J.; Frolov, S. M.; Duine, R. A. New Perspectives for Rashba Spin-Orbit Coupling. Nat. Mater. 2015, 14, 871-882.

(51) Rienks, E. D. L.; Ärrälä, M.; Lindroos, M.; Roth, F.; Tabis, W.; Yu, G.; Greven, M.; Fink, J. High-Energy Anomaly in the Angle-Resolved Photoemission Spectra of Nd2XCexCuO4: Evidence for a Matrix Element Effect. Phys. Rev. Lett. 2014, 113, 137001.

(52) Damascelli, A.; Hussain, Z.; Shen, Z. X. Angle-Resolved Photoemission Studies of the Cuprate Superconductors. Rev. Mod. Phys. 2003, 75, 473-541.

(53) Bistritzer, R.; Khalsa, G.; MacDonald, A. H. Electronic Structure of Doped D0 Perovskite Semiconductors. Phys. Rev. B 2011, 83, 115114.

(54) Oliva, R.; Laurien, M.; Dybala, F.; Kopaczek, J.; Qin, Y.; Tongay, S.; Rubel, O.; Kudrawiec, R. Pressure Dependence of Direct Optical Transitions in ReS2 and ReSe2. npj 2D Mater. Appl. 2019, 3, 20.

(55) Zhao, W.; Ribeiro, R. M.; Eda, G. Electronic Structure and Optical Signatures of Semiconducting Transition Metal Dichalcogenide Nanosheets. Acc. Chem. Res. 2015, 48, 91-99.

(56) Yuan, H.; Liu, X.; Afshinmanesh, F.; Li, W.; Xu, G.; Sun, J.; Lian, B.; Curto, A. G.; Ye, G.; Hikita, Y.; Shen, Z.; Zhang, S. C.; Chen, X.; Brongersma, M.; Hwang, H. Y.; Cui, Y. Polarization-Sensitive Broadband Photodetector Using a Black Phosphorus Vertical p-n Junction. Nat. Nanotechnol. 2015, 10, 707.

(57) Qiao, J.; Kong, X.; Hu, Z. X.; Yang, F.; Ji, W. High-Mobility Transport Anisotropy and Linear Dichroism in Few-Layer Black Phosphorus. Nat. Commun. 2014, 5, 4475.

(58) Kyung, W. S.; Huh, S. S.; Koh, Y. Y.; Choi, K. Y.; Nakajima, M.; Eisaki, H.; Denlinger, J. D.; Mo, S. K.; Kim, C.; Kim, Y. K. Enhanced Superconductivity in Surface-ElectronDoped Iron Pnictide Ba(Fe 1.94 Co 0.06) 2 As 2. Nat. Mater. 2016, 15, 1233-1237.

(59) Katoch, J.; Ulstrup, S.; Koch, R. J.; Moser, S.; McCreary, K. M.; Singh, S.; Xu, J.; Jonker, B. T.; Kawakami, R. K.; Bostwick, A.; Rotenberg, E.; Jozwiak, C. Giant SpinSplitting and Gap Renormalization Driven by Trions in Single-Layer WS2/h-BN Heterostructures. Nat. Phys. 2018, 14, 355-359.

(60) Mucha-Kruczyński, M.; Abergel, D. S. L.; McCann, E.; Fal'Ko, V. I. On Spectral 
Properties of Bilayer Graphene: The Effect of an SiC Substrate and Infrared MagnetoSpectroscopy. J. Phys. Condens. Matter 2009, 21, 344206.

(61) Qiu, Z.; Trushin, M.; Fang, H.; Verzhbitskiy, I.; Gao, S.; Laksono, E.; Yang, M.; Lyu, P.; Li, J.; Su, J.; Telychko, M.; Watanabe, K.; Taniguchi, T.; Wu, J.; Castro Neto, A. H.; Yang, L.; Eda, G.; Adam, S.; Lu, J. Giant Gate-Tunable Bandgap Renormalization and Excitonic Effects in a 2D Semiconductor. Sci. Adv. 2019, 5, 2347.

(62) Ugeda, M. M.; Bradley, A. J.; Shi, S. F.; Da Jornada, F. H.; Zhang, Y.; Qiu, D. Y.; Ruan, W.; Mo, S. K.; Hussain, Z.; Shen, Z. X.; Wang, F.; Louie, S. G.; Crommie, M. F. Giant Bandgap Renormalization and Excitonic Effects in a Monolayer Transition Metal Dichalcogenide Semiconductor. Nat. Mater. 2014, 13, 1091-1095.

(63) Gao, S.; Yang, L. Renormalization of the Quasiparticle Band Gap in Doped TwoDimensional Materials from Many-Body Calculations. Phys. Rev. B 2017, 96, 155410.

(64) Liang, Y.; Yang, L. Carrier Plasmon Induced Nonlinear Band Gap Renormalization in Two-Dimensional Semiconductors. Phys. Rev. Lett. 2015, 114, 063001.

(65) Yao, K.; Yan, A.; Kahn, S.; Suslu, A.; Liang, Y.; Barnard, E. S.; Tongay, S.; Zettl, A.; Borys, N. J.; Schuck, P. J. Optically Discriminating Carrier-Induced Quasiparticle Band Gap and Exciton Energy Renormalization in Monolayer MoS2. Phys. Rev. Lett. 2017, $119,087401$.

(66) Zhang, Y.; Ugeda, M. M.; Jin, C.; Shi, S.; Bradley, A. J.; Mart, A.; Ryu, H.; Kim, J.; Tang, S.; Kim, Y.; Zhou, B.; Hwang, C.; Chen, Y.; Wang, F.; Crommie, M. F.; Hussain, Z.; Shen, Z.; Mo, S. Electronic Structure, Surface Doping, and Optical Response in Epitaxial WSe 2 Thin Films. Nano Lett. 2016, 16, 2485-2491.

(67) Nguyen, P. V.; Teutsch, N. C.; Wilson, N. P.; Kahn, J.; Xia, X.; Graham, A. J.; Kandyba, V.; Giampietri, A.; Barinov, A.; Constantinescu, G. C.; Yeung, N.; Hine, N. D. M.; Xu, X.; Cobden, D. H.; Wilson, N. R. Visualizing Electrostatic Gating Effects in TwoDimensional Heterostructures. Nature 2019, 572, 220.

(68) Jiang, S.; Hong, M.; Wei, W.; Zhao, L.; Zhang, N.; Zhang, Z.; Yang, P.; Gao, N.; Zhou, X.; Xie, C.; Shi, J.; Huan, Y.; Tong, L.; Zhao, J.; Zhang, Q.; Fu, Q.; Zhang, Y. Direct Synthesis and In Situ Characterization of Monolayer Parallelogrammic Rhenium Diselenide on Gold Foil. Commun. Chem. 2018, 1, 17.

(69) Zhang, Z.; Ji, X.; Shi, J.; Zhou, X.; Zhang, S.; Hou, Y.; Qi, Y.; Fang, Q.; Ji, Q.; Zhang, Y.; Hong, M.; Yang, P.; Liu, X.; Zhang, Q.; Liao, L.; Jin, C.; Liu, Z.; Zhang, Y. Direct Chemical Vapor Deposition Growth and Band-Gap Characterization of MoS2/h-BN van Der Waals Heterostructures on Au Foils. ACS Nano 2017, 11, 4328-4336.

(70) Miwa, J. A.; Ulstrup, S.; Sørensen, S. G.; Dendzik, M.; Čabo, A. G.; Bianchi, M.; Lauritsen, J. V.; Hofmann, P. Electronic Structure of Epitaxial Single-Layer MoS2. Phys. Rev. Lett. 2015, 114, 165422.

(71) Bruix, A.; Miwa, J. A.; Hauptmann, N.; Wegner, D.; Ulstrup, S.; Grønborg, S. S.; Sanders, C. E.; Dendzik, M.; Grubišić Čabo, A.; Bianchi, M.; Lauritsen, J. V.; Khajetoorians, A. A.; Hammer, B.; Hofmann, P. Single-Layer MoS2 on Au(111): Band Gap Renormalization and Substrate Interaction. Phys. Rev. B 2016, 93 (16), 1-10.

(72) Moser, S. An Experimentalist's Guide to the Matrix Element in Angle Resolved Photoemission. J. Electron Spectros. Relat. Phenomena 2017, 214, $29-52$.

(73) Chang, Y. J.; Bostwick, A.; Kim, Y. S.; Horn, K.; Rotenberg, E. Structure and Correlation Effects in Semiconducting SrTiO3. Phys. Rev. B - Condens. Matter Mater. Phys. 2010, 81, 235109.

(74) Giannozzi, P.; Baroni, S.; Bonini, N.; Calandra, M.; Car, R.; Cavazzoni, C.; Ceresoli, 
D.; Chiarotti, G. L.; Cococcioni, M.; Dabo, I.; Dal Corso, A.; De Gironcoli, S.; Fabris, S.; Fratesi, G.; Gebauer, R.; Gerstmann, U.; Gougoussis, C.; Kokalj, A.; Lazzeri, M.; Martin-Samos, L.; Marzari, N.; Mauri, F.; Mazzarello, R.; Paolini, S.; Pasquarello, A.; Paulatto, L.; Sbraccia, C.; Scandolo, S.; Sclauzero, G.; Seitsonen, A. P.; Smogunov, A.; Umari, P.; Wentzcovitch, R. M. QUANTUM ESPRESSO: A Modular and Open-Source Software Project for Quantum Simulations of Materials. J. Phys. Condens. Matter 2009, 21, 395502.

(75) P Giannozzi , O Andreussi, T Brumme, O Bunau, M. B.; Nardelli, M Calandra, R Car, C Cavazzoni, D Ceresoli, M. C.; N Colonna, I Carnimeo, A Dal Corso, S. de G.; P Delugas, R A DiStasio Jr, A Ferretti, A Floris, G. F.; G Fugallo, R Gebauer, U Gerstmann, F Giustino, T Gorni, J. J.; M Kawamura, H-Y Ko , A Kokalj, E Küçükbenli, M. L.; M Marsili, N Marzari, F Mauri, N L Nguyen, H.-V. N.; A Otero-de-la-Roza, L Paulatto, S Poncé, D Rocca, R. S.; B Santra, M Schlipf, A P Seitsonen, A Smogunov, I. T.; T Thonhauser, P Umari, N Vast, X. W. and S. B. Advanced Capabilities for Materials Modelling with Q Uantum ESPRESSO. J. Phys. Condens. Matter 2017, 29, 465901.

(76) Dal Corso, A. Pseudopotentials Periodic Table: From H to Pu. Comput. Mater. Sci. 2014, 95, 337-350.

(77) Perdew, J. P.; Zunger, A. Self-Interaction Correction to Density-Functional Approximations for Many-Electron Systems. Phys. Rev. B 1981, 23, 5048-5079.

(78) Perdew, J. P.; Burke, K.; Ernzerhof, M. Generalized Gradient Approximation Made Simple. Phys. Rev. Lett. 1996, 77, 3865-3868.

(79) Fletcher, D. A.; McMeeking, R. F.; Parkin, D. The United Kingdom Chemical Database Service. J. Chem. Inf. Comput. Sci. 1996, 36, 746-749.

(80) Monkhorst, H. J.; Pack, J. D. Special Points for Brillouin-Zone Integrations*. Phys. Rev. B 1976, 13, 5188-5192. 


\section{Supporting Information}

\section{Visualizing Orbital Content of Electronic Bands in Anisotropic}

\section{D Semiconducting ReSe2}

Byoung Ki Choi, ${ }^{1}$ Søren Ulstrup, ${ }^{2,3}$ Surani M. Gunasekera, ${ }^{4}$ Jiho Kim, ${ }^{5}$ Soo Yeon Lim, ${ }^{6}$ Luca Moreschini, ${ }^{3}$ Ji Seop Oh, ${ }^{3,7,8}$ Seung-Hyun Chun, ${ }^{9}$ Chris Jozwiak, ${ }^{3}$ Aaron Bostwick, ${ }^{3}$ Eli Rotenberg, ${ }^{3}$ Hyeonsik Cheong, ${ }^{6}$ In-Whan Lyo, ${ }^{5}$ Marcin Mucha-Kruczynski, ${ }^{4}$ and Young Jun Chang,,$^{* *}$

${ }^{1}$ Department of Physics, University of Seoul, Seoul 02504, Republic of Korea

${ }^{2}$ Department of Physics and Astronomy, Aarhus University, Denmark, 8000 Aarhus C, Denmark

${ }^{3}$ Advanced Light Source (ALS), E. O. Lawrence Berkeley National Laboratory, Berkeley, California 94720, USA

${ }^{4}$ Centre for Nanoscience and Nanotechnology and Department of Physics, University of Bath, Bath BA2 7AY, United Kingdom

${ }^{5}$ Department of Physics, Yonsei University, Seoul, 03722, Republic of Korea

${ }^{6}$ Department of Physics, Sogang University, Seoul, 04107, Republic of Korea

${ }^{7}$ Center for Correlated Electron Systems, Institute for Basic Science (IBS), Seoul 08826, Republic of Korea

${ }^{8}$ Department of Physics and Astronomy, Seoul National University, Seoul 08826, Republic of Korea

${ }^{9}$ Department of Physics, Sejong University, Seoul 05006, Republic of Korea

*e-mail: yjchang@uos.ac.kr 


\section{2D character of valence band structure in ML ReSe2}

Figure S1 shows photon energy $(h v)$-dependence of the valence band structure in ML $\mathrm{ReSe}_{2}$ for both p- and s-polarized light configurations. Energy and momentum positions of the bands do not change for different $h v$ values, although their relative intensities vary due to different cross-sections or geometric effects. The lack of dispersion with $h v$ is consistent with the 2D nature of the electronic structure in the single-layer limit.
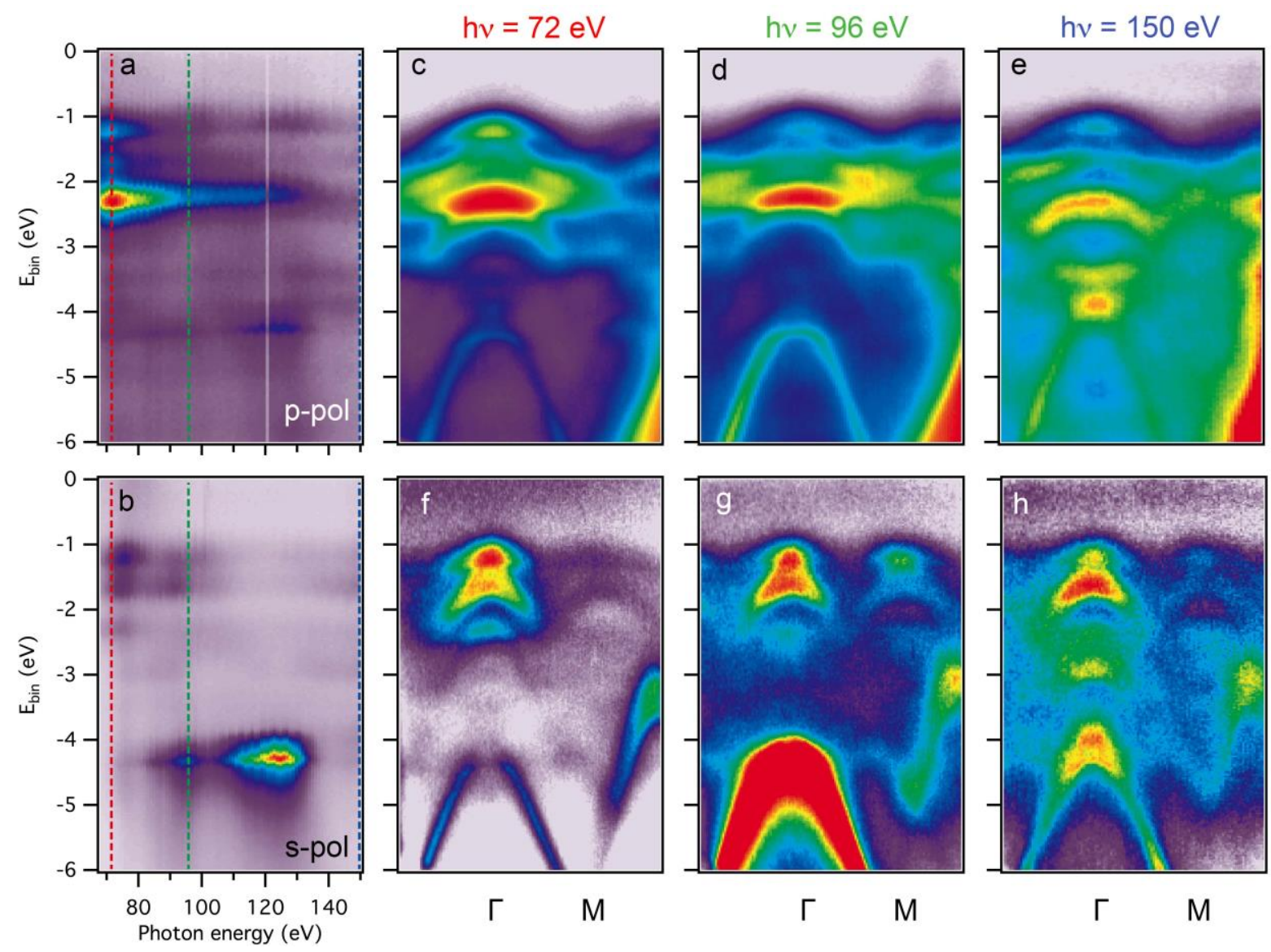

Figure S1. 2D character of valence band structure in ML $\operatorname{ReSe}_{2}$. (a,b) Photon energy $(h v)$ dependence of valence band cuts at $\Gamma$ with p- (a) and s-polarized light (b). (c-e) Valence bands along $\Gamma$-M for $h v=72$ (c), 96 (d), $150 \mathrm{eV}$ (e) using p-polarization. (f-h) Valence bands along $\Gamma-\mathrm{M}$ for $h v=72$ (f), $96(\mathrm{~g}), 150 \mathrm{eV}(\mathrm{h})$ using s-polarization. The 2D electronic structure of the ML $\operatorname{ReSe}_{2}$ is confirmed by the lack of dispersion with $h v$. 


\section{Symmetric band structure of ML ReSe2 along three equivalent axes}

Figure $\mathrm{S} 2$ shows symmetric valence band structure of $\mathrm{ML} \mathrm{ReSe}_{2}$ along the principal axes $(\Gamma-$ $\mathrm{M}_{\mathrm{i}}$ and $\Gamma-\mathrm{K}_{\mathrm{i}}$ for $\left.\mathrm{i}=1,2,3\right)$. The band cuts display similar features along each of the $\Gamma-\mathrm{M}_{\mathrm{i}}$ or $\Gamma-$ $\mathrm{K}_{\mathrm{i}}$ directions, showing their effective equivalence.
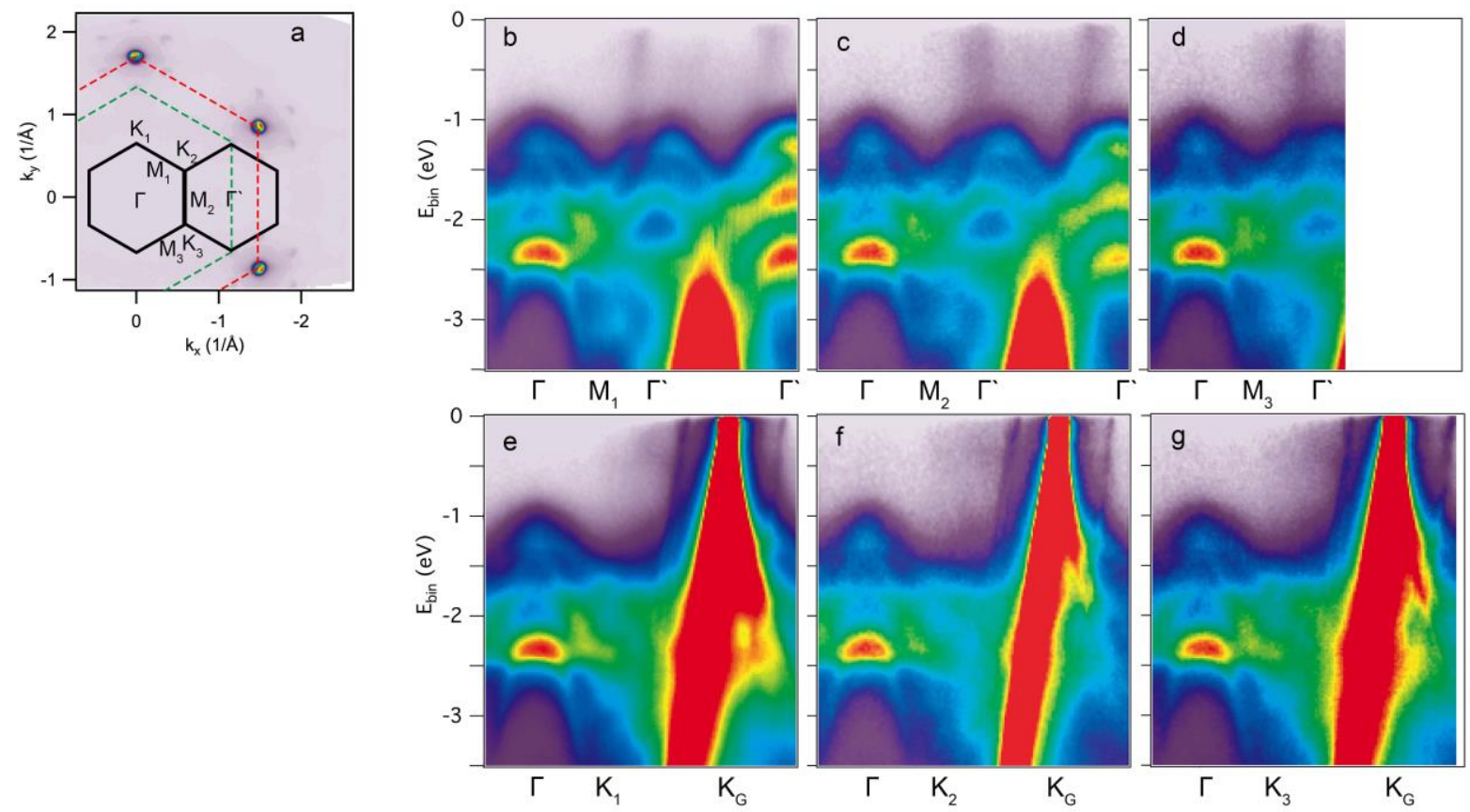

Figure S2. Symmetric valence band structure of ML $\mathrm{ReSe}_{2}$ along the principal axes. (a) Fermi surface map of ML ReSe 2 (b-g) Band cuts along $\Gamma-\mathrm{M}_{1}$ (b), $\Gamma-\mathrm{M}_{2}$ (c), $\Gamma-\mathrm{M}_{3}(\mathrm{~d}), \Gamma-\mathrm{K}_{1}$ (e), $\Gamma-\mathrm{K}_{2}$ (f), and $\Gamma-\mathrm{K}_{3}(\mathrm{~g})$ directions. (All spectra her were obtained with the p-polarization and $h v=96$ eV.) 


\section{Valence band maximum analysis during potassium deposition}

Figure S3 presents an energy distribution curve (EDC) analysis of a polycrystalline Au spectrum and the $\mathrm{ReSe}_{2}$ valence band maximum (VBM) during potassium deposition. The EDC extracted from the Au spectrum is fitted by a Fermi-Dirac distribution. VBM fits of $\mathrm{ReSe}_{2}$ consist of two components (VB1 and VB2) that are described by gaussian functions.
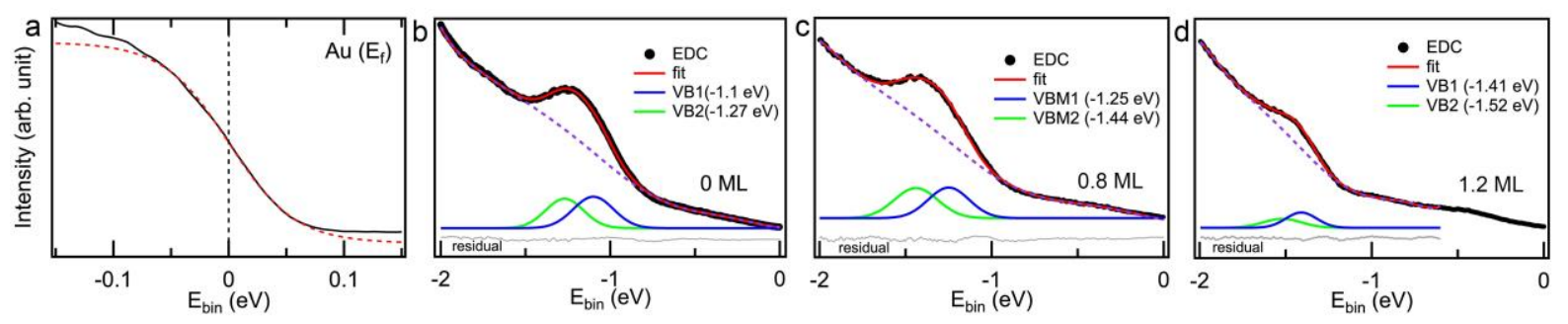

Figure S3. VBM shift during potassium deposition. (a) EDC around the Fermi edge taken on polycrystalline Au in order to calibrate the energy scale. (b-d) VBM fits of ML $\operatorname{ReSe}_{2}$ spectra for a potassium coverage of $0 \mathrm{ML}$ (b), $0.8 \mathrm{ML}$ (c), and 1.2 ML (d). 


\section{Temperature dependence of valence band structure in ML $\operatorname{ReSe}_{2}$ and BLG}

Figure S4 presents a comparison between the ARPES spectra taken at sample temperatures of $80 \mathrm{~K}$ and $270 \mathrm{~K}$. During the temperature cycle, we observe $\sim 50 \mathrm{meV}$ energy shift of the $\mathrm{ReSe}_{2}$ VBM, which is a relatively small change. This small shift implies that a temperature-induced change of carrier density does not significantly alter the electronic structure of ML $\operatorname{ReSe}_{2}$ on BLG. We also note that all the measurements were performed at $\sim 90 \mathrm{~K}$, so that the temperature effect is minimal for understanding the rest of ARPES results.
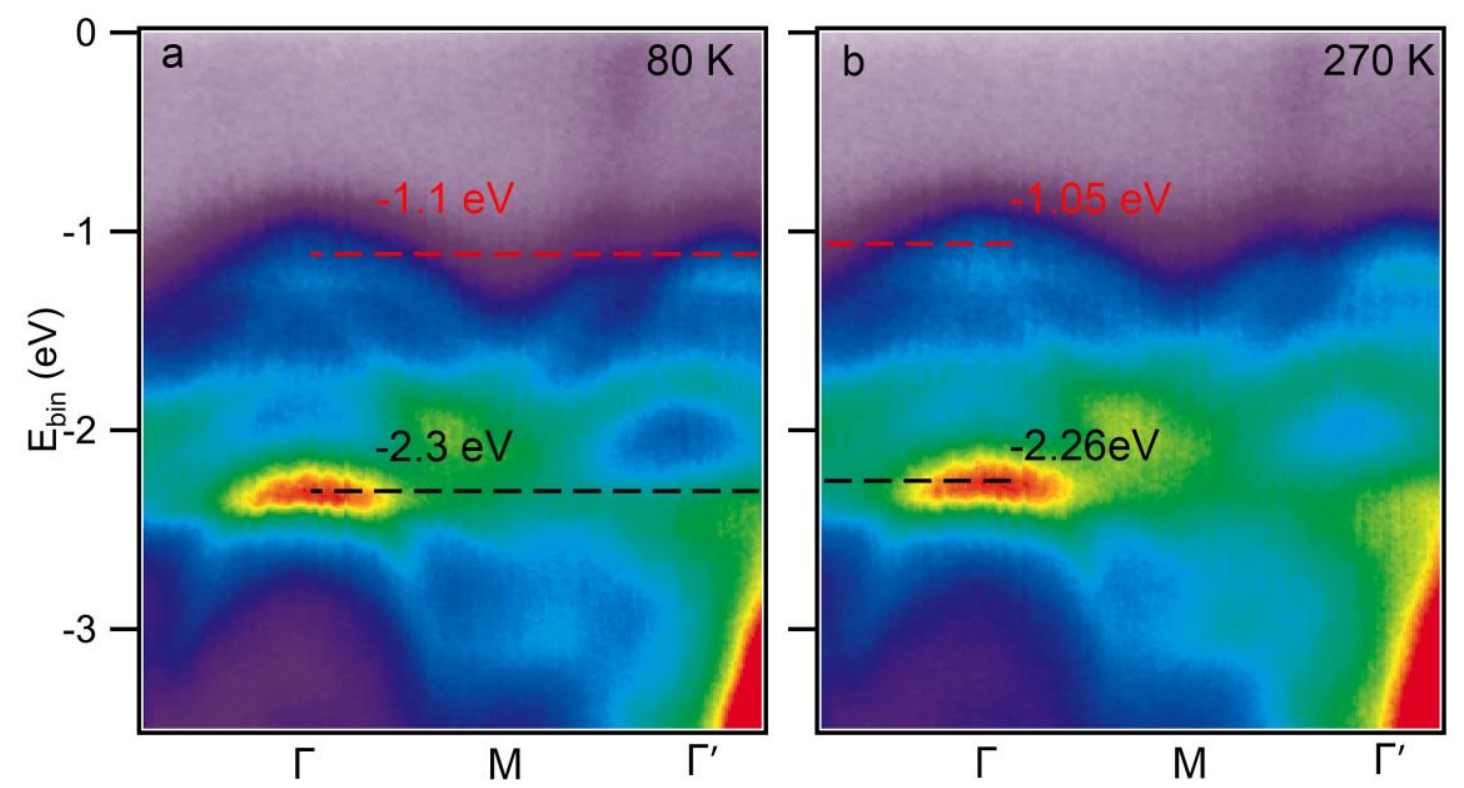

Figure S4. Temperature dependence of valence band structure in ML ReSe 2. (a,b) ARPES spectra for ML $\operatorname{ReSe}_{2}$ along $\Gamma-\mathrm{M}-\Gamma^{\prime}$ at $80 \mathrm{~K}$ (a) and $270 \mathrm{~K}$ (b). 


\section{Orbital character of valence bands}

Figure S5 presents the orbital-projected valence band calculation for a wide momentum range. These orbital projected bands show qualitative agreement with the ARPES spectra in Fig. 3ad.
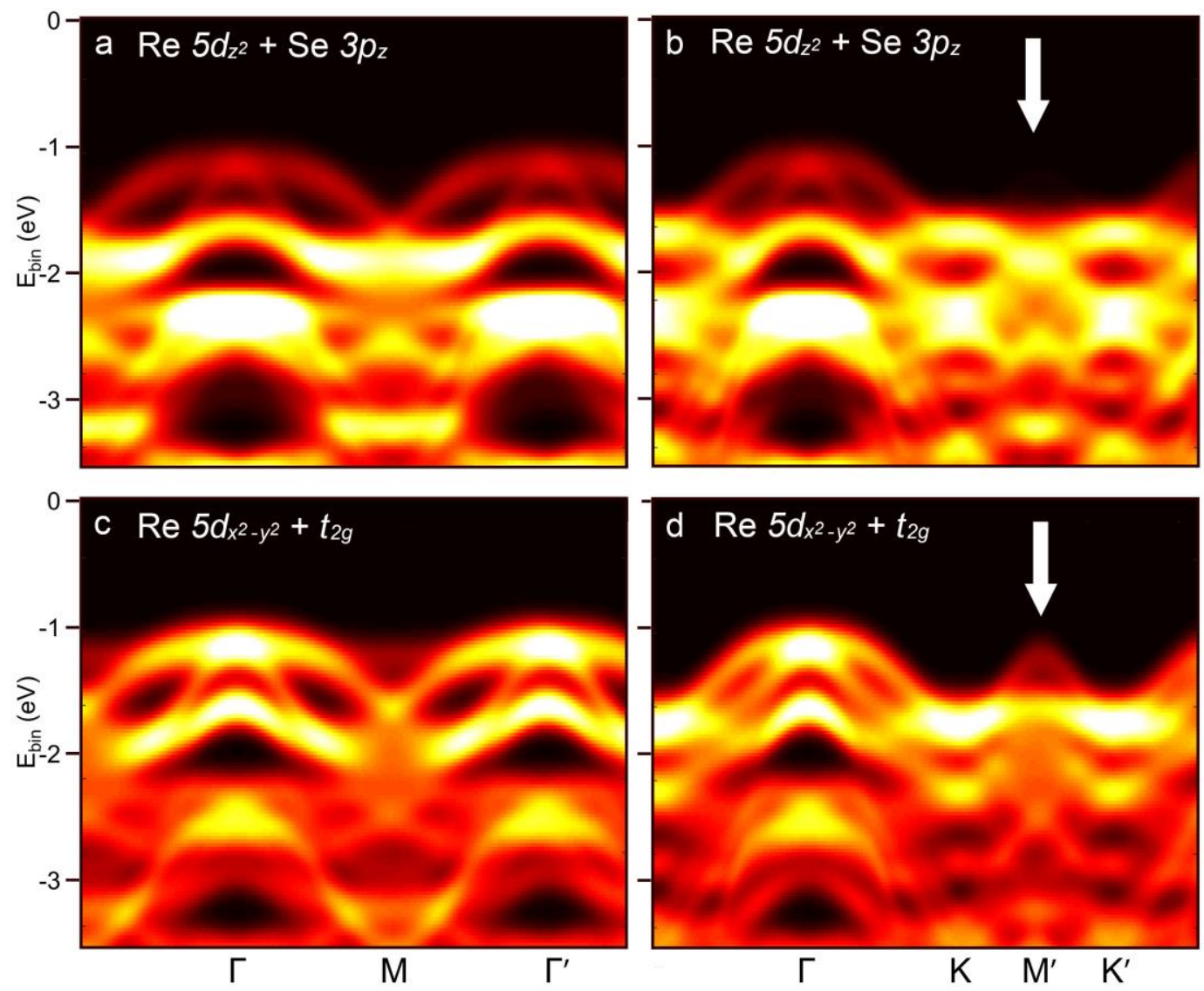

Figure S5. Orbital-projected valence band density of states in ML ReSe2. (a,b) Contribution of $\operatorname{Re} 5 d_{z}{ }^{2}$ and Se $3 p_{z}$ orbitals along $\Gamma-\mathrm{M}-\Gamma^{\prime}$ (a) and $\Gamma-\mathrm{K}-\mathrm{M}^{\prime}(\mathrm{b})$. (c,d) Contribution of $\operatorname{Re} d_{\mathrm{x} 2-\mathrm{y} 2}$ and $t_{2 \mathrm{~g}}$ orbitals along $\Gamma-\mathrm{M}-\Gamma^{\prime}(\mathrm{c})$ and $\Gamma-\mathrm{K}-\mathrm{M}^{\prime}(\mathrm{d})$. The white arrows in (b) and (d) indicate the intensity close to the $\mathrm{M}^{\prime}$ point from electronic states of one of the domains also marked in Fig. 3. 


\section{Surface electron doping via potassium deposition}

Figure S6 shows X-ray photoemission spectroscopy (XPS) data of potassium ( $3 p$ ) and rhenium $(4 f)$ collected during surface electron doping via potassium deposition. The potassium $3 p$ spectra exhibit a systematic intensity increase with potassium coverage. When the second potassium layer forms, an interface peak emerges on the left of the main surface peak. We define potassium density at which the interface peak emerges as one monolayer (1 ML) coverage. During the potassium deposition, the rhenium $4 f$ spectra show an energy shift of $0.32 \mathrm{eV}$ as seen in Fig. S6d, which is consistent with the VBM shift $(-0.3 \mathrm{eV})$ discussed in the main manuscript. It is noted that the small shoulders (Re-Se) on the right side of main peaks are probably caused by the defect phase formed during Se-decapping. These shoulders do not shift during the potassium deposition.
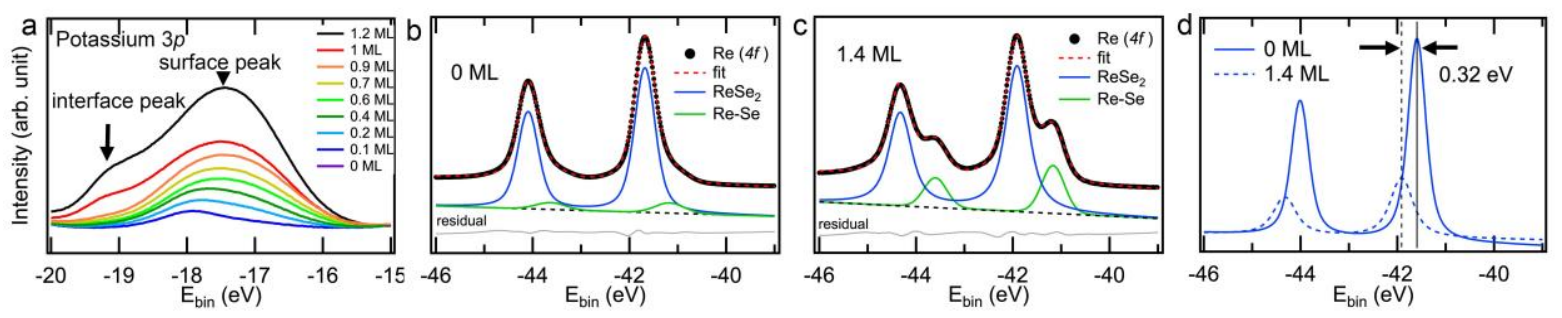

Figure S6. Surface electron doping via potassium deposition. (a) XPS data of potassium 3p. (bd) XPS data of rhenium $4 f$ at a coverage of 0 ML (b) and 1.4 ML (c). (d) Comparison of the main peaks $\left(\mathrm{ReSe}_{2}\right)$ for the two coverages. 


\section{Doping dependence of valence band structure in ML ReSe2 and BLG}

Figure S7 presents the valence bands of $\mathrm{ReSe}_{2}$ and bilayer graphene for different surface potassium doping levels in order to compare the respective band alignments. In the pristine sample, the charge neutrality point of the BLG is given by $E_{N}=-0.33 \mathrm{eV}$ below the Fermi level. In comparison, VBM of $\mathrm{ReSe}_{2}$ is located at $E_{V B M}=-1.1 \mathrm{eV}$ below the Fermi level. At 1.2 ML potassium coverage, the $E_{N}$ shifts to $-0.53 \mathrm{eV}$ below the Fermi level while the VBM moves to $E_{V B M}=-1.41 \mathrm{eV}$. During the doping, the 2D carrier density of BLG changes from $1.6 \times 10^{13}$ $\mathrm{cm}^{-2}$ to $4.1 \times 10^{13} \mathrm{~cm}^{-2}$ (we estimate these carrier densities by using BLG density of states as provided by a standard tight-binding model), which corresponds to $2.1 \times 10^{13} \mathrm{~cm}^{-2}$ per ML potassium coverage. Since this carrier density only accounts for the doping of the BLG, the total contributing carrier density from the potassium should also include the doping of ML $\mathrm{ReSe}_{2}$.
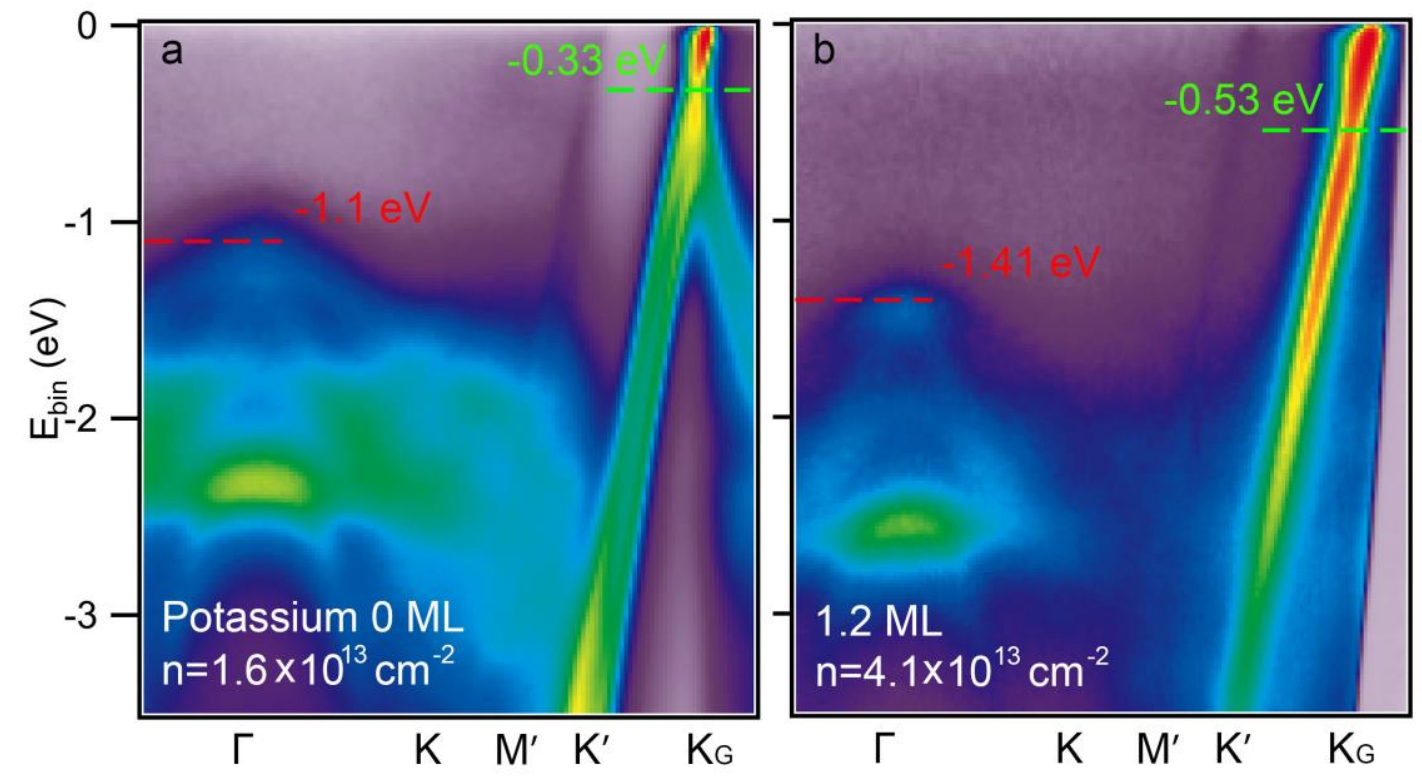

Figure S7. Doping dependence of valence band structure in ML ReSe $e_{2}$ and BLG. (a,b) ARPES spectra for ML $\operatorname{ReSe}_{2}$ along $\Gamma-\mathrm{K}-\mathrm{M}^{\prime}$ for potassium coverage of $0 \mathrm{ML}$ (a) and 1.2 ML (b). Red and green dashed lines indicate the VBM of ML $\operatorname{ReSe}_{2}$ and the neutrality point $\left(E_{N}\right)$ of BLG, respectively. 